\title{
CATÁLOGO PRELIMINAR DE LAS PLANTAS UASCULARES DE LOS BOSQUES DEL CORREDOR BIOLÓGICO FORMADO ENTRE LOS PARQUES NACIONALES NATURALES PURACÉ Y CUEUA DE LOS GUÁCHAROS (HUILA, COLOMBIA)
}

Palabras clave: Bosque andino, Biodiversidad, Catálogo florístico, Colombia, Corredor Biológico. Key words: Andean Forest, Biodiversity, Biologic Corridor, Colombia, Floristic catalogue.

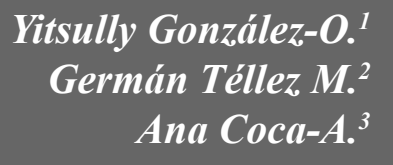

Yitsully González-O. ${ }^{1}$

Ana Coca-A. ${ }^{3}$

\section{RESUMEN}

Se presenta un catálogo de las especies encontradas en los bosques pertenecientes al corredor biológico ubicado entre los Parques Nacionales Naturales (PNN) Puracé, Cueva de los Guácharos, al sur del departamento del Huila, Colombia. El objetivo del catálogo es identificar las especies de las áreas boscosas y perturbadas del corredor. El muestreo de especies se realizó durante el levantamiento de vegetación en un área total de 1475 ha necesaria para caracterizar florística y estructuralmente los bosques del corredor biológico. El listado es una muestra de las especies concentradas en los bosques subandinos y andinos mejor conservados y de las áreas intervenidas entre 1900 y 2443 m de altitud para tres de los cuatro municipios que hacen parte del corredor biológico: San Agustín, Pitalito y Acevedo. Se encontraron 274 especies de plantas vasculares distribuidas en 82 familias, siendo las más ricas Rubiaceae, Lauraceae y Melastomataceae. Para el presente estudio se reportaron
266 especies de Angiospermas, 7 especies de Pteridófitos y una Gimnosperma, el pino colombiano Retrophyllum rospigliosii.

\begin{abstract}
A catalogue of the plant species found in the forests of the biological corridor located between the National Parks Puracé and Cueva de los Guácharos, Southern Huila Department, Colombia is presented. The main goal of the catalogue was to identify the species of the woodlands and disturbed areas in the biological corridor. Species sampling was made in a 1475 ha area during a vegetation survey made in order to characterize the flora and the structure of the biological corridor forests. The list is a representative sample of the species found in both conserved and disturbed Andean and sub-Andean forests between 1900 and $2443 \mathrm{~m}$ of altitude belonging to three of the four municipalities of the corridor: San Agustín, Pitalito and Acevedo. We found 274 vascular species
\end{abstract}

\footnotetext{
Ingeniera Forestal. Asistente de Investigacion, Herbario Forestal UDBC, Universidad Distrital "Francisco Jose de Caldas", Bogota, Colombia. tisú11y2@hotmail.com

2 Ingeniero Forestal. Curador Herbario Forestal UDBC, Universidad Distrital "Francisco Jose de Caldas", Bogota, Colombia. germantmc@ gmail.com

3 Ingeniera Forestal. Asistente de Investigacion, Herbario Forestal UDBC, Universidad Distrital "Francisco Jose de Caldas”, Bogotá, Colombia. anitakro07@hotmail.com
} 
of 82 plant families. The richest families were Rubiaceae, Lauraceae, and Melastomataceae. A total number of 266 species for Angiospermae, 7 species for Pterydophyta and only one Gimnospermae, the Colombian pine, Retrophyllum rospigliosii, were registered in this study.

\section{INTRODUCCIÓN}

En las áreas que hacen parte del corredor biológico se hallan diversas zonas de vida. En el Parque Nacional Natural Puracé, se encuentran páramos y una serie de lagunas que son conocidas como la "estrella fluvial de Colombia" de gran importancia hidrológica puesto que en su conjunto son el nacimiento de los ríos Patía, Magdalena, Cauca, Caquetá y Putumayo. Igualmente a lo largo del corredor biológico se localizan bosques altoandinos, andinos y subandinos con bajo grado de intervención. Así mismo se encuentran extensas áreas destinadas a actividades agrícolas y pecuarias, que también requieren un manejo apropiado para evitar que se extiendan hacia zonas que no tienen esa aptitud.

En los bosques andinos colombianos se han realizado diversos estudios tendientes a conocer aspectos tales como su composición florística (Galindo et al. 2003, Giraldo 1995) su fisionomía (Rangel \& Lozano 1986), su diversidad (Phillips \& Miller 2002, Rangel 1995) y fitosociología (Cleef et al. 1984). Una visión general de los aspectos físicos y biológicos de los bosques andinos en Colombia se presenta en Andrade (1993) y Cavelier et al. (2001).

Aunque la mayoría de los bosques del corredor biológico entre los PNN Puracé y Cueva de los Guácharos presenta una alta diversidad y un relativo grado de conservación, son muy pocos los trabajos que se han realizado para conocer la vegetación existente. Se conocen estudios descriptivos y sobre la composición florística como el realizado por Cleef (1972), quien elaboró un informe inicial sobre la flora del Parque Nacional Natural Cueva de los Guácharos; Bernal (1973) quien realizó un estudio estructural en el municipio de Acevedo; Lozano et al. (1979) y Hernández et al. (1980), quienes registraron por primera vez la presencia de la especie Colombobalanus excelsa (= Trigonobalanus excelsa) en el Parque Nacional Natural Cueva de los Guácharos. Rangel \& Lozano (1986) realizaron la descripción de la vegetación en una franja desde La Plata (Huila) hasta el volcán del Puracé, y el más reciente estudio fué elaborado por Mahecha (1999) sobre las especies más importantes del sector de Villalobos vía Mocoa - Pitalito. Por lo tanto la Corporación Regional del Alto Magdalena (CAM) aunó esfuerzos con el fin de elaborar un estudio más específico sobre la composición y estructura de la vegetación en los bosques que hacen parte del corredor biológico entre los PNN Puracé y Cueva de los Guácharos; del cual se deriva esta publicación. Por consiguiente, el objetivo del presente trabajo fue elaborar un catálogo preliminar de las especies que se registraron en cada uno de los bosques analizados.

\section{ÁREA DE ESTUDIO}

El corredor biológico entre los Parques Nacionales Naturales Puracé y Cueva de los Guácharos se encuentra ubicado al sur del departamento del Huila entre los $5^{\circ} 57^{\prime} 19$ " a $6^{\circ} 38^{\prime} 17.6^{\prime \prime} \mathrm{N}$ y $\operatorname{los} 72^{\circ} 14^{\prime} 38^{\prime \prime}$ a $73^{\circ} 2$ ' 20" W (Figuras 1 y 2). Se encuentra incluido en los municipios de San Agustín, Pitalito, Acevedo y Palestina en un área de 84.465 ha (CAM 1997).

El catálogo de las especies que se presenta en este trabajo hace parte del estudio florístico y estructural realizado por Coca \& González (2007) en el cual se caracterizó la mayor parte de los bosques que componen el corredor biológico, se realizaron inventarios de vegetación en 16 áreas de muestreo entre 500 y $1000 \mathrm{~m}^{2}$ 


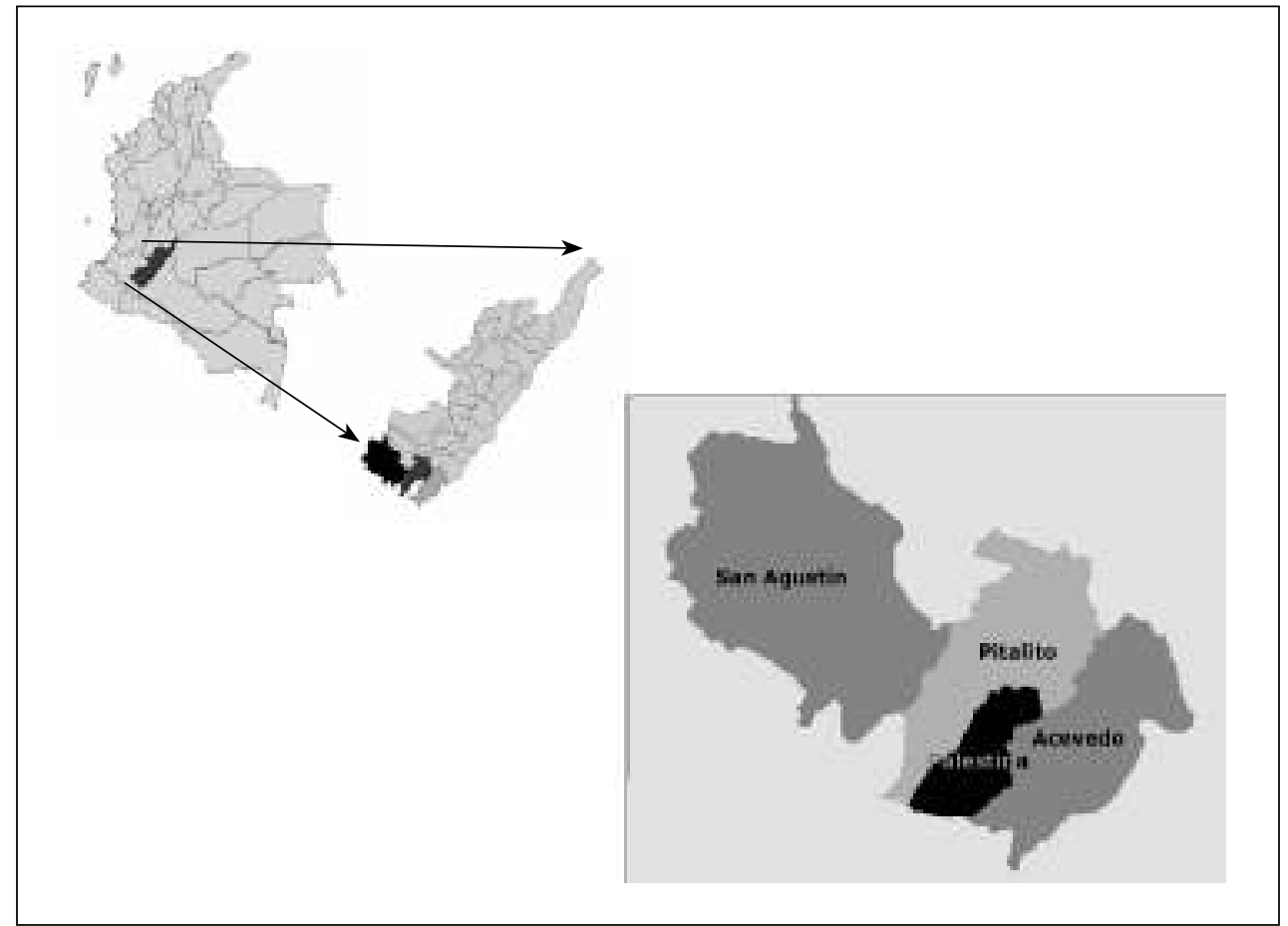

Figura 1. Área de estudio. Ubicación general del corredor biológico entre los PNN Puracé, Cueva de los Guácharos (modificado del mapa de trabajo del proyecto corredor biológico).

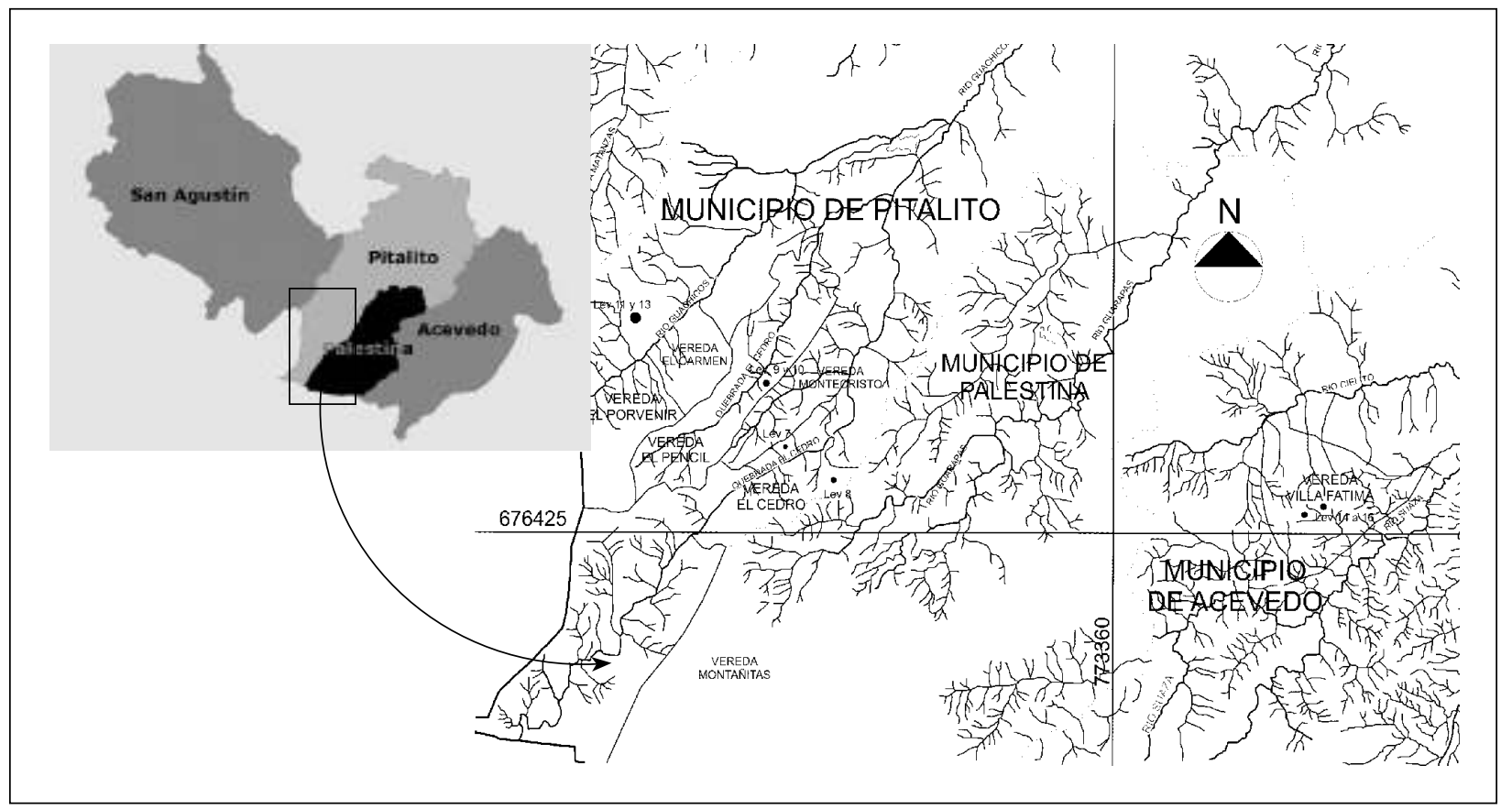

Figura 2. Ubicación de las parcelas realizadas. a. Municipio de San Agustín b. Municipio de Pitalito (Modificado del mapa de trabajo del proyecto corredor biológico y del estudio de Coca \& González, 2007). 
en un área de 1475 ha. Los levantamientos se elaboraron en los municipios de San Agustín, Pitalito y Acevedo. En el municipio de San Agustín se realizaron seis levantamientos en las veredas Villa Fátima (2012 m), Yarumal (2200 m), San Antonio (2277 m) Alto Quinchana (2443 $\mathrm{m}$ ) y Marbella en donde se realizo un levantamiento en bosque $(2120 \mathrm{~m})$ y otro en rastrojo (2055 m)En Pitalito, se realizaron levantamientos en áreas boscosas e intervenidas en las veredas El Cedro (2080 m y $1969 \mathrm{~m}$ ), El Pencil (1900 y 1955 m) y El Porvenir, donde se realizó un levantamiento en bosque $(2102 \mathrm{~m})$ y dos en áreas intervenidas (1989 y 2036 m). En Acevedo se realizaron levantamientos en la vereda Villa Fátima a 2094 m.

De acuerdo a la clasificación climática de Holdridge (1979) las estaciones de Pitalito, San Agustín y Suaza corresponden a la zona de vida bosque húmedo premontano $(\mathrm{Bh}-\mathrm{PM})$. De acuerdo al Sistema de Clasificación de Cuatrecasas (1958) la mayor parte del área se define como Selva Subandina (1900-2400 m) y aquellas regiones que superan los $2400 \mathrm{~m}$ de altitud se definen como Selva Andina.

Según la clasificación de Caldas \& Lang (1918), citado en Eslava et al. (1986a), en las estaciones ubicadas en los municipios de San Agustín (Río Naranjo) y Pitalito se presenta un clima templado semihúmedo, mientras que en la estación de Acevedo (Río Suaza) se describe un clima templado semiárido. De acuerdo al sistema de clasificación de Köeppen (1936), citado en Eslava et al. (1986b), el clima del corredor biológico se clasifica como Gw ó clima de montaña húmedo de tierras templadas y frías.

\section{MÉTODOS}

Como se describe detalladamente en Coca \& González (2007), la vegetación del Corredor Biológico se caracterizó mediante parcelas de
$1000 \mathrm{~m}^{2}$ evaluando individuos con DAP $\geq 10$ $\mathrm{cm}$, subparcelas de $100 \mathrm{~m}^{2}$ para evaluar arbolitos y para la vegetación herbácea subparcelas de $16 \mathrm{~m}^{2}$.

Los especimenes botánicos recolectados fueron procesados en el Herbario Forestal de la Universidad Distrital "Francisco José de Caldas" (UDBC). El proceso de identificación se realizó en el Herbario Forestal (UDBC) y en el Herbario Nacional Colombiano (COL), mediante el uso de literatura y comparación con los especimenes depositados. Los nombres científicos y su respectivo autor fueron estandarizados y abreviados usando las páginas en la Web de IPNI (Internacional Plant Names Index) http://www.ipni.org/ y W $\mathrm{W}^{3}$ Trópicos http:// mobot.mobot.org/.

Adicionalmente, se realizaron algunas colecciones fuera de los levantamientos identificando especimenes pertenecientes a las familias Ericaceae, Podocarpaceae y Polygalaceae.

El catálogo se organizó en tres grandes grupos: Pteridófitos, Gimnospermas y Angiospermas. Las Angiospermas fueron definidas de acuerdo al sistema de clasificacion de Cronquist (1981) en dos subgrupos, Dicotiledóneas (Magnoliopsida) y Monocotiledóneas (Liliopsida). Dentro de estos grupos y subgrupos, las familias se presentan alfabéticamente, al igual que los géneros y las especies. Las familias de Pteridófitos se organizaron alfabéticamente.

Cada especie incluye la siguiente información: categoría de tamaño, estado de conservación o desarrollo del bosque, unidad sintaxonómica definida de acuerdo al estudio realizado por Coca \& González (2007), localidad geográfica (municipio y vereda) y números de colección correspondientes bajo las series de numeración de Carolina Coca (CKA) y Yitsully González (YGO). Para algunas especies, también se incluye el nombre vernáculo. 
La categoría de tamaño, se refiere al rango de diámetro y altura en el cual se encontró la especie en el momento de la colección, conociendo su importancia al situarse en varios estratos a la vez. Se define como brinzal (B) a aquel individuo cuya altura está entre los 0.3 y $1.5 \mathrm{~m}$; latizal (L) para el individuo con altura superior a $1.5 \mathrm{~m}$ y diámetro a la altura del pecho (DAP) menor a $10 \mathrm{~cm}$ y fustal a aquel con diámetro mayor a 10 $\mathrm{cm}$ de DAP. En el caso de plantas no erectas se diferencian los bejucos y plantas trepadoras.

El estado de conservación o desarrollo se definió de acuerdo a los resultados de la caracterización estructural y la composición florística presentada en Coca \& González (2007). En la parte estructural, fue relevante el análisis de los histogramas de frecuencia y área basal en las clases diamétricas y de alturas así como el nivel de estratificación de cada unidad sintaxonómica. En la composición florística, se tuvo en cuenta la presencia de especies esciófitas, secundarias tardías, secundarias iniciales o pioneras. De esta manera, las áreas de acuerdo a su estado de conservación o desarrollo se definieron como bosque poco intervenido, bosque intervenido, bosque secundario de regeneración y rastrojo.

De acuerdo a la caracterización florística y estructural de la vegetación realizada por Coca \& González (2007) se encontraron siete unidades sintaxonómicas: una alianza conformada por tres asociaciones y tres comunidades independientes, las cuales se mencionan con sus respectivas convenciones al inicio del catálogo.

\section{RESULTADOS}

Se encontraron 274 especies de plantas vasculares correspondientes a 82 familias y 163 géneros, siendo las Angiospermas las de mayor riqueza específica con 266, seguida de los Pteridofitos con 7 y las Gimnospermas con una especie.
Dentro de las Angiospermas, las Dicotiledóneas (Magnoliopsida) son el grupo con mayor riqueza con 248 especies representando un $93 \%$ del total, tal como lo muestra la Tabla No. 1.

Tabla 1. Riqueza por grandes grupos taxonómicos para la vegetación del corredor biológico entre los $\mathrm{PNN} \mathrm{Pu-}$ racé, Cueva de los Guácharos.

\begin{tabular}{|l|r|c|c|}
\hline \multirow{2}{*}{\multicolumn{1}{|c|}{ Grupo }} & \multicolumn{3}{|c|}{ Riqueza } \\
\cline { 2 - 4 } & Familias & Géneros & Especies \\
\hline Pteridófitos & 6 & 6 & 7 \\
Gimnospermas & 1 & 1 & 1 \\
Angiospermas Dicotiledóneas & 69 & 143 & 248 \\
Monocotiledóneas & 6 & 13 & 18 \\
\hline
\end{tabular}

Las familias de Angiospermas con mayor número de especies y géneros son Rubiaceae, Lauraceae y Melastomataceae. La familia Asteraceae se destaca por su alta riqueza genérica con 12 géneros. Otras familias sobresalientes fueron Clusiaceae, Piperaceae, Myrsinaceae y Arecaceae (Tabla 2).

Tabla 2. Familias de mayor riqueza genérica y de especies. Corredor biológico entre los PNN Puracé, Cueva de los Guácharos.

\begin{tabular}{|l|c|c|}
\hline \multirow{2}{*}{ Familias } & \multicolumn{2}{|c|}{ Riqueza } \\
\cline { 2 - 3 } & Géneros & Especies \\
\hline Rubiaceae & 15 & 28 \\
Lauraceae & 8 & 23 \\
Melastomataceae & 7 & 23 \\
Asteraceae & 12 & 14 \\
Clusiaceae & 5 & 11 \\
Piperaceae & 2 & 10 \\
Myrsinaceae & 3 & 9 \\
Arecaceae & 6 & 8 \\
\hline
\end{tabular}

Los géneros con más especies fueron Miconia (15), Palicourea (9), Piper (8), Ocotea (7) у

Revista Colombia Forestal Vol. 10 No. 20 - Diciembre 2007 
Clusia (7), todos pertenecientes a las Dicotiledóneas (Magnoliopsida).

Dentro de las categorías de grado de intervención o desarrollo del bosque, el área inventa- riada como bosque poco intervenido fue la que obtuvo mayor riqueza de especies con 192, seguida del área de rastrojo, tal como se muestra en la Tabla 3. Igualmente, la categoría de tamaño "latizal" fue la de mayor riqueza.

Tabla 3. Riqueza de especies por categoría de tamaño y estado de conservación o desarrollo del bosque. Corredor biológico entre los PNN Puracé y Cueva de los Guácharos.

\begin{tabular}{|l|r|r|c|c|c|}
\hline \multicolumn{1}{|c|}{ Categ. de tamaño } & No sps & $\%$ & Estado de Construcción & No sps & $\%$ \\
\hline Brinzal & 134 & 49 & Bosque poco intervenido & 192 & 70 \\
Latizal & 167 & 61 & Bosque intervenido & 11 & 4 \\
Fustal & 125 & 46 & Bosque de regeneración & 35 & 13 \\
Bejuco & 4 & 1 & Rastrojo & 72 & 26 \\
Trepadora & 1 & 0 & & & \\
\hline
\end{tabular}

\section{DISCUSIÓN Y CONCLUSIONES}

Se compararon las familias de mayor riqueza específica del corredor biológico con las registradas en otros estudios elaborados en bosques andinos a altitudes similares, encontrándose varias diferencias en la riqueza de las principales familias, debidas principalmente a la metodología aplicada en la caracterización de dichas áreas. Consideramos que los métodos aplicados en el trabajo realizado por Coca y
González (2007) repercutieron en la ausencia y baja diversidad de familias que predominan en el hábito de crecimiento herbáceo, tales como Orchidaceae y Gesneriaceae que estuvieron entre las más diversas en el PNN Munchique (Lozano et al. 1996), Polypodiaceae y Araceae en el PNN Los Nevados (Rangel 1995) y Orchidaceae y Polypodiaceae en la Plata Huila, Puracé (Rangel \& Lozano 1986), tal como se muestra en la Tabla No. 4.

Tabla 4. Familias con mayor riqueza para distintas localidades de bosque andino en Colombia.

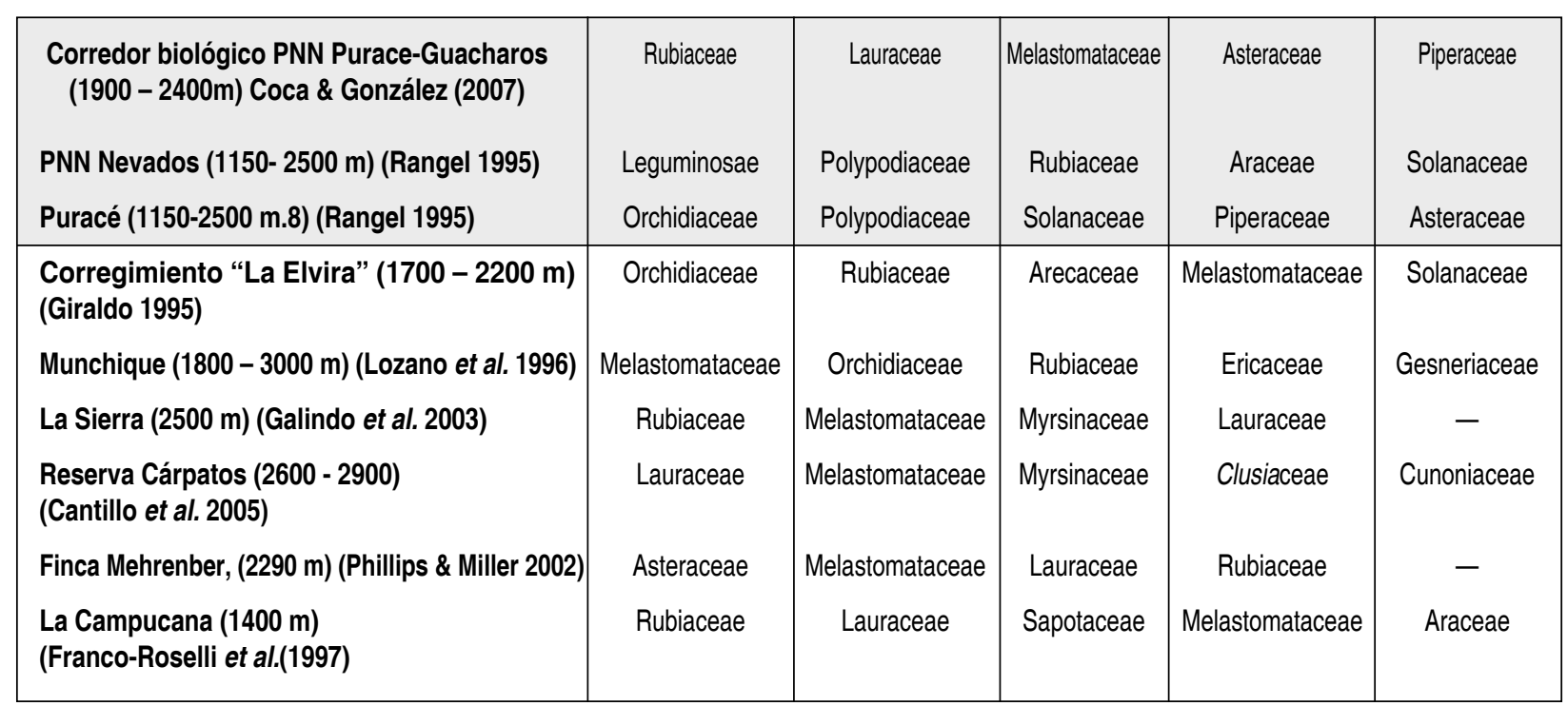


Si realizamos esta misma comparación de riqueza a nivel de familias entre el corredor biológico e inventarios realizados incluyendo solamente individuos con DAP $\geq$ a $1,2.5$, o $10 \mathrm{~cm}$ en los bosques andinos neotropicales a alturas similares, vemos que son notorias las similitudes entre estos. Las tres familias con mayor número de especies encontradas en este estudio son en su orden, Rubiaceae, Lauraceae y Melastomataceae que coinciden con las mencionadas por Gentry (2001) como las más diversas en los bosques andinos entre 1500 y $2500 \mathrm{~m}$. Sin embargo, el orden de las familias no es el mismo. Para Gentry (2001), la familia más diversa es Lauraceae seguida de Rubiaceae y Melastomataceae. Igualmente, Cantillo et al. (2005), Franco-Roselli et al. (1997), Galindo et al. (2003) y el muestreo de 0,1 ha realizado por Gentry (Phillips \& Miller, 2002) en la Finca Mehrenberg (Huila) cerca de la zona de estudio (Tabla No. 4) mencionan a las mismas familias como las de mayor importancia a nivel de número de especies, con leves diferencias debidas a la altitud del muestreo. Otras familias importantes para el corredor biológico como Clusiaceae, Euphorbiaceae, Solanaceae también son mencionadas por Gentry (2001) y Giraldo (1995) como importantes en bosques andinos de elevaciones medias.

La riqueza de especies a nivel de géneros nos muestra que Miconia, es el género más rico. Este género es mencionado por Galeano (1994) como un elemento común en áreas con cierto grado de intervención, aunque algunas de sus especies se destacan en el bosque primario. De igual forma, Gentry (2001) menciona a Miconia como el genero más diverso tanto en bosques montanos andinos como centroamericanos, seguido de Ocotea, Piper y Palicourea, lo cual concuerda también con lo obtenido en este estudio.

Otros géneros notablemente diversos en Puracé y en el PNN Los Nevados (Rangel 1995) como Peperomia y Solanum (con más de 20 especies cada uno) presentaron menos de cinco especies en este estudio.

De manera preliminar, se reporta para el corredor biológico la especie endémica del sur del departamento del Huila Perrottetia guachara$n a$, y como novedad corológica para la zona se reporta la especie Matudaea colombiana.

Se concluye que los bosques del corredor biológico entre los PNN Puracé y Cueva de los Guácharos no presentan diferencias en su composición florística con la de los bosques andinos colombianos en lo que se refiere a las familias y géneros de mayor riqueza.

De acuerdo al inventario florístico elaborado en el corredor biológico se deduce que aún se conservan áreas relativamente amplias de gran riqueza florística con especies endemicas del sur del departamento del Huila como Perrottetia guacharana y especies de importancia ecológica, como Quercus humboldtii y Tocoyena costanensis importantes para el mantenimiento de especies de fauna silvestre como el oso andino Tremarctos ornatus y la danta de montaña Tapirus pinchaque que son de importancia regional.

Consideramos que la flora vascular y principalmente la flora arbórea y arbustiva del corredor biológico entre los PNN Puracé y Cueva de los Guácharos, presenta una alta riqueza a nivel de familias y especies, pese a que este trabajo es la etapa inicial en el estudio y conocimiento de la vegetación de dicha región.

\section{AGRADECIMIENTOS}

Queremos agradecer por el financiamiento a la CAM, en especial a Gloria Ramírez, quien nos brindó su conocimiento y experiencia en campo y a todos los integrantes del equipo técnico y profesional del corredor biológico; a Edgard E. Cantillo por la dirección del proyecto; al 
Herbario Forestal de la Universidad Distrital "Francisco José de Caldas" (UDBC), en especial a su directora Rocío Cortés-B. y al Herbario Nacional Colombiano (COL) de la Universidad Nacional de Colombia por facilitar el trabajo de determinación de los especimenes.

\section{REFERENCIAS BIBLIOGRÁFICAS}

Andrade, G. 1993. Carpanta: Selva Nublada y Páramo, Fundación Natura-Colombia. Bogotá.

Bernal, A. 1973. Estudio de la vegetación y planificación preliminar del Parque Nacional Natural Cueva de los Guácharos, Acevedo, Huila. Trabajo de Grado, Facultad del Medio Ambiente y Recursos Naturales. Universidad Distrital Francisco José de Caldas. Bogotá D.C., Colombia.

Cantillo, E., A. Avella, \& K. Rodriquez 2005. La vegetación leñosa de la reserva foresta Cárpatos. Universidad Distrital Francisco José de Caldas. Bogotá.

Cavelier, J., D. Lizcano \& M. Pulido. 2001. Colombia. Pgs 444 - 496. en: Kappelle M. \& A. Brown (eds). Bosques nublados del Neotrópico. Instituto Nacional de Biodiversidad (INBio), Santo Domingo de Heredia.

Cleef, A. 1972. Informe preliminar sobre la botánica del PNN Cueva de los Guácharos. Bogotá. Colombia.

Cleef, A., J.O. Rangel, T. Van Der Hammen \& R. Jaramillo. 1984. La vegetación de las selvas del transecto Buritaca. Pgs. 267 - 406. En Van Der Hammen, T. \& P. Ruiz (eds.). Estudios de ecosistemas tropandinos. J. Cramer. Berlín, Sttutgart.

Coca, C. \& Y. González. 2007. Caracterización florística y estructural de la vegetación del Corredor Biológico entre los Parques Nacionales Naturales Puracé - Cueva de los Guácharos. 282 pg. Bogotá D.C., Colombia. Trabajo de grado (Ingeniero Forestal). Universidad Distrital Francisco José de Caldas.

Corporación Autónoma Regional del Alto Magdalena (CAM). 1997. Agenda ambiental del municipio de San Agustín. 67 pg.

Cronquist, A. 1981. An integrated system of classification of flowering plants. Columbia University Press. New York.

Cuatrecasas, J. 1958. Aspectos de la vegetación natural de Colombia. Rev. Acad. Colomb. Cienc. 10(40): 221-268.

Eslava, J.V. López \& O. Olaya. 1986a. Los Climas de Colombia. Sistemas de Caldas \& Lang (1918) Atmósfera (5) Pgs. 35 - 81. Sociedad Colombiana de Meteorología, Santafé de Bogotá.

Eslava, J., V. López \& O. Olaya. 1986b. Los Climas de Colombia. Sistema de W. Köeppen (1936) Atmósfera (7) Pgs. 41 - 77. Sociedad Colombiana de Meteorología, Santafé de Bogotá.

Franco-Rosselli, P., J. Betancur \& J. L. Fernández-Alonso. 1997. Diversidad florística en dos bosques subandinos del sur de Colombia. Caldasia 19 (1-2): 205-234.

Galeano, M. P. 1994. Composición florística del Parque Regional Natural Ucumari. 451 pg. en: J. O. Rangel (ed.) Ucumarí un caso típico de la diversidad biotica andina.

Galindo-T., J. Betancur \& J. Cadena-M. 2003. Estructura y Composición Florística de cuatro bosques andinos del Santuario de Flora y Fauna Guanentá-Alto Río Fonce, 
Cordillera oriental colombiana. Caldasia 25 (2): $313-335$.

Gentry, A. 2001. Patrones de diversidad y composición florística en los bosques de las montañas neotropicales. Pgs. 85- 123. En: Kappelle M. \& A. Brown (eds). Bosques nublados del Neotrópico. Instituto Nacional de Biodiversidad (INBio), Santo Domingo de Heredia.

Giraldo, J. 1995. La Flora de un bosque montano húmedo en la Cordillera Occidental. Cespedesia 20 (66): 181-186.

Hernández-C., J., G. Lozano-C. J. E. Henao-S. 1980. El género Trigonobalanus en el Neotrópico, II. Caldasia 13: 9 - 43.

Holdridge, L. R. 1979. Ecología basada en zonas de vida. Costa Rica. Publicaciones del IICA.

Lozano-C., G., J. Hernández-C y J. E. Henao-S 1979. El género Trigonobalanus en el Neotrópico, I. Caldasia 12: 517 - 537.

Lozano-C. G., N. Ruiz, F. González \& M. T. Murillo. 1996. Diversidad vegetal del Parque Nacional Natural Munchique, Cauca
(Colombia entre 1800 y $3050 \mathrm{msnm}$ ). Pgs 9 - 64. en: P Pinto. Notas sobre biodiversidad. Instituto de Ciencias Naturales - Museo de Historia Natural. Biblioteca José Jerónimo Triana No. 13.

Mahecha, G. E. 1999. Estudio Florístico de la Región del Macizo Colombiano. Principios y Procedimientos para el conocimiento de las plantas superiores del trópico americano. Convenio Intercorporativo del Macizo Colombiano. Huila, Colombia.

Phillips, O. \& J. Miller. 2002. Global Patterns of Plant Diversity: Alwin Gentry's Forest Transect Data Set. Missouri Botanical Garden Press.

Rangel-Ch., O. 1995. La diversidad florística en el espacio andino de Colombia. En: $\mathrm{S}$ Churchill., P. Baslev, E. Forero, J. Luteyn (eds.). Biodiversity and Conservation of Neotropical Montane Forests. The New York Botanical Garden. 187 - 205 pp.

Rangel-Ch., O. \& G. Lozano-C., 1986. Un perfil de vegetación entre La Plata (Huila) y el volcán del Puracé. Caldasia 14: 503 $-547$. 


\section{ANEXO 1}

Catálogo preliminar de las plantas vasculares presentes en el corredor biológico entre los Parques Nacionales Naturales Puracé y Cueva de los Guácharos

El catálogo se divide en tres grandes grupos: Pteridófitos, Gimnospermas y Angiospermas y dentro de estas, las Dicotiledóneas y las Monocotiledóneas. La información de la especie contiene la(s) categoría(s) de tamaño en la(s) que se encontró la especie, el estado de conservación o desarrollo del bosque, las unidades sintaxonómicas, el municipio, la vereda y los números de colección. Las convenciones utilizadas para nombrar las unidades sintaxonómicas son las siguientes:

- As L-E Asociación Ladenbergio macrocarpae - Elaeagietum myrianthae

- As. W-C Asociación Weinmannio pubescentis - Clusietum dixonii

- As. G-M Asociación Guettardo hirsutae - Miconietum sp.

- Com. H - A Comunidad de Helicostylis tovarensis y Helicostylis tovarensis

- $\quad$ Com. Q - WComunidad de Quercus humboldtii y Wettinia fascicularis

- Com. B - S Comunidad de Baccharis nitida y Saurauia pulchra

\section{PTERIDÓFITOS}

Blechnaceae (1 género, 1 especie)

Blechnum divergens (Kze.) Mett.

Brinzal; bosque poco intervenido; As. L - E. San Agustín, vereda Yarumal, CKA 37.

\section{Dennstaedtiaceae (1 género, 1 especie) Dennstaedtia sp.}

Brinzal; bosque poco intervenido; As. L - E. San Agustín, vereda Yarumal, YGO 167.

\section{Grammitidaceae (1 género, 1 especie)}

Terpsichore pichinchae (Sodiro) A. R. Sm. Brinzal; bosque poco intervenido; As. W - C. San Agustín, vereda Alto Canelo, YGO 65.

\section{Hymenophyllaceae (1 género, 1 especie)} Hymenophyllum fucoides (Sw.) Sw.

Brinzal; bosque poco intervenido; As. L - E. San Agustín, vereda Alto Canelo, YGO 66.

\section{Marattiaceae (1 género, 1 especie)}

Danaea aff. cuspidata Liebm.

Brinzal; bosque poco intervenido; As. L - E. San Agustín, vereda Yarumal, YGO 165.

\section{Pteridaceae (1 género, 2 especies)}

Pteris podophylla $\mathrm{Sw}$.

Brinzal; bosque poco intervenido; As. L - E. San Agustín, vereda Alto Canelo, YGO 63.

Pteris speciosa Mett.

Brinzal; bosque poco intervenido; As. W - C, Com. H - A. San Agustín, vereda Alto Canelo, YGO 64.

\section{GIMNOSPERMAS}

Podocarpaceae (1 género, 1 especie) Retrophyllum rospigliosii (Pilg.) C.N. Page Nombre vernáculo: "pino colombiano" Latizal; bosque intervenido. Pitalito, vereda El Cedro, YGO 330.

\section{ANGIOSPERMAS}

\section{DICOTILEDÓNEAS}

Acanthaceae (1 género, 1 especie)

Aphelandra cf. runcinata Klotzsch ex Nees Brinzal; bosque poco intervenido; As. L - E. San Agustín, vereda Yarumal, CKA 14. 


\section{Actinidiaceae (1 género, 2 especies)}

Saurauia aff. caquetensis. R. E. Schult. Brinzal, latizal; rastrojo; Com. B - S. Pitalito, vereda El Cedro, CKA 208.

\section{Saurauia pulcra Sprague}

Nombre vernáculo: "moquillo"

Brinzal, latizal, fustal; bosque poco intervenido, bosque intervenido, rastrojo; As. L - E, Com. B - S, Com. Q -W. Pitalito, veredas El Cedro, El Pencil, El Porvenir, Acevedo, vereda Villa Fátima, CKA 202, CKA 248.

\section{Anacardiaceae (1 género, 1 especie)}

Tapirira guianensis Aubl.

Nombre vernáculo: "fresno"

Fustal; bosque poco intervenido; As. L - E, Com. Q -W. San Agustín, vereda Yarumal, Acevedo, vereda Villa Fátima, YGO 442.

\section{Annonaceae (2 géneros, 2 especies)}

Guatteria cf. goudotiana Triana \& Planch.

Latizal; bosque poco intervenido; Com. Q-W. Acevedo, vereda Villa Fátima, CKA 310.

\section{Rollinia sp.}

Nombre vernáculo: "nauino"

Latizal, fustal; bosque poco intervenido; As. L - E, Com. H - C, Com. Q - W. San Agustín, vereda Yarumal, Pitalito, vereda El Cedro, CKA 33, CKA 186.

\section{Apocynaceae (1 género, 1 especie)} Mandevilla $\mathrm{cf}$. veraguensis Hemsl.

Trepadora; rastrojo; As. G - M. San Agustín, vereda Los Alpes - Marbella, YGO 277.

\section{Aquifoliaceae (1 género, 1 especie)}

Ilex laurina Kunth

Fustal, bosque poco intervenido; Com. $\mathrm{H}-\mathrm{C}$, Com. Q - W. Pitalito, vereda El Pencil, Acevedo, vereda Villa Fátima, CKA 235, YGO 448.

\section{Araliaceae ( 2 géneros, 5 especies)}

Oreopanax incisum (Willd. ex Schult.) Decne. \& Planch.
Brinzal, latizal; bosque intervenido, rastrojo; As. G - M, Com. B - S. San Agustín, vereda Los Alpes - Marbella, Pitalito, vereda El Pencil, CKA 237, YGO 243.

Oreopanax ruizii Decne. \& Planch. ex Harms Latizal, fustal; bosque poco intervenido; Com. $\mathrm{H}$ - A. Pitalito, vereda El Cedro, CKA 171, YGO 315.

Schefflera cf. ciliata Cuatrec.

Latizal; bosque poco intervenido; Com. Q - W. Acevedo, vereda Villa Fátima, CKA 309.

Schefflera cf. sphaerocoma (Benth.) Harms Brinzal, latizal; bosque poco intervenido, bosque en regeneracion; Com. $\mathrm{H}-\mathrm{A}, \mathrm{Com} . \mathrm{Q}-\mathrm{W}$. San Agustín, vereda Villa Fátima, Pitalito, vereda El Pencil, Acevedo, vereda Villa Fátima, CKA 92, YGO 387, YGO 387b.

Schefflera quindiuensis (Kunth) Harms Brinzal, latizal bosque poco intervenido. As. W - C, Com. Q - W. San Agustín, vereda Alto Canelo, Acevedo, vereda Villa Fátima, CKA 278, YGO 58.

\section{Asteraceae (12 géneros, 14 especies)}

Austroeupatorium inulifolium (Kunth) R.M. King \& H. Rob.

Nombre vernáculo: "salvia"

Brinzal, latizal; rastrojo; As. G - M, Com. B - S. San Agustín, vereda Marbella, Pitalito, vereda El Porvenir, CKA 141.

Baccharis cf. decussata (Klatt) Hieron. Brinzal; bosque intervenido; Com. Q - W. Acevedo, vereda Villa Fátima, CKA 281.

Baccharis latifolia (Ruiz \& Pav.) Pers. Latizal; rastrojo; As. G - M. San Agustín, vereda Marbella, CKA 137b.

Baccharis nitida (Ruiz \& Pav.) Pers. Nombre vernáculo: "chilco blanco" 
Brinzal, latizal, fustal; rastrojo; Com. B - S. San Agustín, vereda Marbella, Pitalito, veredas El Cedro, El Pencil, El Porvenir, CKA 146.

Chromolaena cf. odorata (L.) R. M. King \& H. Rob.

Nombre vernáculo: "chilco negro/rojo" Brinzal, latizal; rastrojo; As. G - M, Com. B - S. San Agustín, vereda Marbella, Pitalito, veredas El Cedro, El Pencil, CKA 137.

Clibadium cf. surinamense L.

Brinzal; rastrojo; Com. B - S. Pitalito, vereda E1 Porvenir, CKA 256.

Condylopodium cuatrecasasii R.M King \& H. Rob.

Brinzal; bosque poco intervenido; Com. $\mathrm{H}-\mathrm{A}$. Pitalito, vereda El Cedro, YGO 308.

Erato vulcanica (Klatt) H. Rob.

Latizal; rastrojo; Com. B - S. Pitalito, veredas El Cedro, El Pencil, El Porvenir, YGO 343.

Liabum asclepiadeum Sch. Bip.

Brinzal; rastrojo; Com. B - S. Pitalito, vereda El Cedro, YGO 334.

Mikania aff. houstoniana (L.) B. L. Rob.

Brinzal; bosque poco intervenido; As. L - E. San Agustín, vereda San Antonio, CKA 10.

Montanoa quadrangularis Sch. Bip. ex K. Koch

Nombre vernáculo: "jiquimillo"

Latizal, fustal; bosque intervenido, rastrojo; As. G - M, Com. B - S. San Agustín, vereda Los Alpes - Marbella, Pitalito, vereda El Pencil, CKA 70, CKA 247.

Munnozia senecionidis Benth.

Brinzal, latizal; bosque poco intervenido, rastrojo; As. G - M, Com. B - S, Com. Q - W. San Agustín, vereda Marbella, Pitalito, vereda El Cedro, Acevedo, vereda Villa Fátima, CKA 200.
Oligactis cf. volubilis (Kunth) Cass.

Brinzal; rastrojo; Com. B - S. Pitalito, vereda El Cedro, YGO 347.

Vernonanthura patens (Kunth) H. Rob.

Latizal; rastrojo; Com. B - S. Pitalito, vereda El Pencil, CKA 240.

\section{Begoniaceae (1 género, 1 especie)}

Begonia cf. maurandiae A. DC.

Brinzal; bosque poco intervenido; As. W - C. San Agustín, vereda Alto Canelo, YGO 61.

\section{Bombacaceae (1 género, 1 especie)}

Spirotheca rhodostyla Cuatrec.

Nombre vernáculo: "tachuelo"

Latizal, fustal; bosque poco intervenido; As. L - E, As G - M, Com. H - A. San Agustín, vereda Los Alpes - Marbella, Pitalito, veredas El Cedro, El Pencil, El Porvenir, CKA 120, CKA 229, CKA 318.

\section{Boraginaceae (1 género, 1 especie) \\ Cordia aff. rubescens Estrada}

Nombre vernáculo: "guacimo"

Fustal; bosque poco intervenido, bosque intervenido; As. L - E, As. G - M, Com B - S. San Agustín, veredas Yarumal, Los Alpes - Marbella, Pitalito, veredas El Pencil, El Porvenir, CKA 246.

\section{Campanulaceae ( 2 géneros, 2 especies)}

Burmeistera cf. succulenta Karst.

Latizal; bosque de regeneración; As. L - E. San Agustín, vereda Villa Fátima, CKA 109.

Centropogon semperflorens E. Wimm

Latizal; rastrojo; Com. B - S. Pitalito, vereda El Pencil, YGO 358.

\section{Capparaceae (1 género, 1 especie)}

Podandrogyne cf. brachycarpa (DC.) Woodson

Brinzal; bosque intervenido; As. G - M. San Agustín, vereda Los Alpes - Marbella, YGO 222. 


\section{Caprifoliaceae (1 género, 4 especies)}

Viburnum cornifolium Killip \& A.C. Sm.

Nombre vernáculo: "pepe-pava"

Fustal; bosque poco intervenido, bosque intervenido; As. G - M, As. W - C. San Agustín, veredas Alto Canelo, Los Alpes - Marbella, CKA 115, CKA 121, YGO 146.

Viburnum glabratum Kunth

Fustal; bosque poco intervenido; As. W - C. San Agustín, vereda Alto Canelo, YGO 142.

Viburnum cf. lehmannii Killip \& A.C. Sm. Brinzal, fustal; bosque poco intervenido; As. L - E, Com. H - A. Pitalito, veredas El Cedro, El Porvenir, CKA 164, CKA 268.

\section{Viburnum sp.}

Nombre vernáculo: "carbonero" "pepe-pava" Brinzal, latizal, fustal; bosque poco intervenido, bosque intervenido, bosque de regeneración, rastrojo; As. L - E, Com. B - S. San Agustín, vereda Yarumal, Pitalito, vereda El Pencil, CKA 59, CKA 243, YGO 7.

\section{Cecropiaceae (1 género, 1 especie)}

Cecropia angustifolia Trécul

Fustal; bosque intervenido, bosque de regeneración, rastrojo; As. L- E, As. G - M, Com. $\mathrm{H}-\mathrm{A}$, Com. B - S. San Agustín, veredas Yarumal, Villa Fátima, Los Alpes - Marbella, Pitalito, vereda El Porvenir, CKA 82, YGO 323.

\section{Celastraceae (1 género, 1 especie)}

Perrottetia guacharana R Sánchez \& Fern. Alonso

Fustal; bosque poco intervenido; Com. $\mathrm{H}$ - A. Pitalito, vereda El Cedro, CKA 190.

\section{Chloranthaceae (1 género, 4 especies)}

Hedyosmum cuatrecazanum Occhioni

Nombre vernáculo: "granicillo" "chiflador"

Brinzal, latizal, fustal; bosque poco intervenido, bosque intervenido, bosque de regenera- ción; As. L - E, As W - C As. G - M, Com. H - A. San Agustín, veredas Alto Canelo, Yarumal, Villa Fátima, Pitalito, veredas El Cedro, El Porvenir, CKA 19, CKA 265, CKA 316, YGO 138, YGO 316.

Hedyosmum goudotianum Solms

Nombre vernáculo: "chiflador"

Brinzal, latizal; bosque poco intervenido; Com. H - A, Com. Q - W. Pitalito, vereda El Pencil, Acevedo, vereda Villa Fátima, CKA 311, YGO 384.

Hedyosmum racemosum (Ruiz \& Pav.) G. Don

Latizal, fustal; bosque poco intervenido, bosque intervenido, bosque de regeneración; As. L - E, As. G - M, Com. Q - W. San Agustín, veredas Villa Fátima, Los Alpes - Marbella, Acevedo, vereda Villa Fátima, CKA 100, YGO 297.

Hedyosmum translucidum Cuatrec.

Brinzal, latizal, fustal; bosque poco intervenido, bosque intervenido, bosque de regeneración; As. $\mathrm{L}-\mathrm{E}$, As. $\mathrm{G}-\mathrm{M}$, Com. $\mathrm{B}-\mathrm{S}$, Com Q - W. San Agustín, vereda Villa Fátima, Pitalito, veredas El Pencil, El Porvenir, Acevedo, vereda Villa Fátima, CKA 84, CKA 117, CKA 170.

\section{Clethraceae (1 género, 1 especie)}

Clethra fagifolia Kunth

Fustal; bosque poco intervenido, bosque de regeneración; As. L-E, As. W - C. San Agustín, veredas Alto Canelo, Villa Fátima, CKA 87, YGO 141.

\section{Clusiaceae (5 géneros, 11 especies)}

Calophyllum aff. brasiliense Cambess.

Brinzal, latizal, fustal; bosque poco intervenido, bosque intervenido; As. G - M, Com. H - A. San Agustín, vereda Los Alpes - Marbe1la, Pitalito, vereda El Pencil, CKA 135, YGO 238, YGO 251, YGO 376. 
Chrysochlamys colombiana (Cuatrec.) Cuatrec. Latizal, fustal; bosque poco intervenido; As. W - C. San Agustín, vereda Alto Canelo, YGO 95, YGO 124.

Clusia cf. alata Planch. \& Triana

Latizal; bosque poco intervenido; Com. Q-W. Acevedo, vereda Villa Fátima, CKA 329.

Clusia discolor Cuatrec.

Fustal; bosque de regeneración; As. L-E. San Agustín, vereda Villa Fátima, CKA 72.

Clusia dixonii Little

Brinzal, fustal; bosque poco intervenido; As. W - C, Com. B - S. San Agustín, vereda Alto Canelo, Pitalito, vereda El Cedro, CKA 61, YGO 344.

Clusia ducuoides Engl

Latizal; bosque poco intervenido; As. W - C. San Agustín, vereda Alto Canelo, YGO 115.

Clusia aff. flavida (Benth.) Pipoly

Fustal; bosque poco intervenido; Com. Q - W. Acevedo, vereda Villa Fátima, CKA 306.

Clusia cf. haughtii Cuatrec.

Latizal; bosque de regeneración; As. L - E. San Agustín, vereda Villa Fátima, YGO 200, YGO 203.

\section{Clusia sp.}

Brinzal, latizal; bosque poco intervenido; As. L - E, As. W - C, As. G - M. San Agustín, veredas Alto Canelo, Los Alpes - Marbella, Pitalito, veredas El Cedro, El Pencil, El Porvenir, CKA 167, YGO 91, YGO 305.

Tovomita weddeliana Planch. \& Triana

Fustal; bosque poco intervenido; Com. Q - W. Acevedo, vereda Villa Fátima, CKA 288.

Vismia baccifera Planch. \& Triana Brinzal, latizal, fustal; bosque poco intervenido, bosque intervenido, bosque de regenera- ción, rastrojo; As. L - E, Com. H - A, Com B - S. San Agustín, vereda Villa Fátima, Pitalito, veredas El Cedro, El Porvenir, CKA 26, YGO 314, YGO 422.

\section{Cornaceae (1 género, 1 especie)}

Cornus peruviana J. F. Macbr.

Fustal; bosque poco intervenido; As. L - E. San Agustín, veredas San Antonio, YGO 8.

\section{Cucurbitaceae (1 género, 1 especie) \\ Rytidostylis sp. 1}

Brinzal; bosque poco intervenido; As. L - E, Com. H - A. San Agustín, vereda Yarumal, Pitalito, vereda El Cedro, CKA 12, CKA 155.

\section{Cunoniaceae (1 género, 4 especies)}

Weinmannia magnifolia Cuatrec.

Nombre vernáculo: "encenillo"

Brinzal, latizal, fustal; bosque poco intervenido, bosque de regeneración; As. L - E, Com. H - A, Com Q - W. San Agustín, veredas Yarumal, Villa Fátima, Pitalito, vereda El Cedro, Acevedo, vereda Villa Fátima, YGO 16.

Weinmannia pubescens Kunth

Nombre vernáculo: "encino"

Fustal; bosque poco intervenido; As. L-E, As. W - C. San Agustín, veredas San Antonio, Alto Canelo, CKA 86, CKA 130, YGO 130.

Weinmannia sorbifolia var. sclerophylla (Cuatrec.) Cuatrec.

Nombre vernáculo: "encenillo"

Fustal; bosque de regeneración; As. L - E. San Agustín, vereda Villa Fátima, CKA 74, CKA 80

Weinmannia subsessiliflora Ruiz \& Pav. subsp. caquetana (Cuatrec.) Bernardi Nombre vernáculo: "encenillo" Latizal, fustal; bosque poco intervenido; As. L - E, As. W - C. San Agustín, veredas San Antonio, Alto Canelo, YGO 16. 


\section{Ericaceae (4 géneros, 5 especies)}

Cavendishia cf. macrocephala A.C. Sm.

Bejuco; San Agustín, vereda Los Alpes - Marbella, CKA 270.

Macleania stricta A.C. Sm.

Bejuco; San Agustín, vereda Los Alpes - Marbella, CKA 271.

Psammisia aff. sclerantha A.C. Sm.

Bejuco; San Agustín, vereda San Antonio, YGO 33, YGO 183.

Psammisia aff. sodiroi Hoerold

Brinzal, latizal; bosque poco intervenido, bosque de regeneración; As. L-E, As. W - C. San Agustín, veredas San Antonio, Alto Canelo, Villa Fátima, YGO 49, YGO 112, YGO 183.

Thibaudia aff. rigidiflora A.C. Sm. Bejuco; San Agustín, vereda Villa Fátima, YGO 187.

\section{Euphorbiaceae (4 géneros, 7 especies)}

Acalypha macrostachya Jacq.

Brinzal, latizal; bosque poco intervenido, rastrojo; As. L - E, Com. B - S. San Agustín, vereda Yarumal, Pitalito, veredas El Cedro, El Porvenir, CKA 54, YGO 335, YGO 406.

Alchornea coelophylla Pax \& K. Hoffm.

Fustal; bosque poco intervenido; Com. $\mathrm{H}$ - A. Pitalito, vereda El Cedro, YGO 324.

Alchornea glandulosa Poepp.

Brinzal, latizal, fustal; bosque poco intervenido; As. L - E, As. W - E. Com. H - A, Com. Q - W. San Agustín, vereda Alto Canelo, Pitalito, veredas El Cedro, El Porvenir, Acevedo, vereda Villa Fátima, CKA 317, YGO 99, YGO 321, YGO 412.

Alchornea grandiflora Müll. Arg.

Latizal, fustal; bosque poco intervenido; As. W - C, Com. H - A, Com. Q - W. San Agustín, vereda Alto Canelo, Acevedo, vereda Villa
Fátima, Pitalito, vereda El Pencil, YGO 136, YGO 160, YGO 428.

Alchornea aff. latifolia Sw.

Nombre vernáculo: "ortigo"

Fustal; bosque poco intervenido; As. L-E, As. G - M. San Agustín, vereda Los Alpes - Marbella, Pitalito, vereda El Porvenir, CKA 216, CKA 269.

Hieronyima huilensis Cuatrec.

Latizal, fustal; bosque poco intervenido; As. W - C, Com. H - A, Com. Q - W. San Agustín, vereda Alto Canelo, Pitalito, vereda El Cedro, Acevedo, vereda Villa Fátima, CKA 172, CKA 174, CKA 181, YGO 84, YGO 157, YGO 447.

Tetrorchidium euryphyllum Standl.

Latizal; bosque poco intervenido; Com. Q - W. Acevedo, vereda Villa Fátima, YGO 439.

Fabaceae ( 2 géneros, 3 especies)

Erythrina edulis Triana ex M. Micheli

Nombre vernáculo: "chachafruto" "balú"

Latizal, fustal; rastrojo; Com. B - S. Pitalito, vereda El Pencil, CKA 245.

\section{Ormosia revoluta Rudd}

Nombre vernáculo: "chirimoyo"

Latizal, fustal; bosque de regeneración; As. L E. San Agustín, vereda Villa Fátima, CKA 76. Ormosia tovarensis Pittier Latizal, fustal; bosque poco intervenido. Com. $\mathrm{H}$ - A, Com. Q - W. Pitalito, vereda El Cedro, Acevedo, vereda Villa Fátima, CKA 327.

\section{Fagaceae (2 géneros, 2 especies)}

Colombobalanus excelsa (Lozano, Hern. Cam. \& Henao) Nixon \& W. L. Crepet

Brinzal, latizal, fustal; bosque poco intervenido. As. L -E, Com. H - A, Com. Q - W. Pitalito, veredas El Pencil, El Porvenir, Acevedo, vereda Villa Fátima, CKA 286, CKA 296, YGO 365. 
Quercus humboldtii Bonpl.

Brinzal, latizal, fustal; bosque poco intervenido; Com. H - A, Com. Q - W. Pitalito, veredas El Cedro, El Pencil, Acevedo, vereda Villa Fátima, CKA 215, CKA 165.

\section{Flacourtiaceae (1 género, 2 especies)}

Casearia cf. mollis Kunth

Fustal; bosque poco intervenido; Com $\mathrm{H}$ - A. Pitalito, vereda El Pencil, CKA 230.

Casearia sylvestris Sw.

Latizal, fustal; bosque poco intervenido, rastrojo; Com. B - S, Com. H - A. Pitalito, vereda El Pencil, CKA 213, CKA 234.

\section{Gesneriaceae (1 género, 3 especies)}

Besleria fecunda C.V. Morton

Brinzal; rastrojo; Com. B - S. Pitalito, vereda El Cedro, YGO 337.

Besleria reticulata Fritsch

Brinzal, latizal; bosque poco intervenido, bosque intervenido, bosque de regeneración; As. L - E, As. W - C, Com. H - A, Com. Q - W. San Agustín, veredas San Antonio, Alto Canelo, Villa Fátima, Pitalito, veredas El Cedro, El Pencil, El Porvenir, Acevedo, vereda Villa Fátima, CKA 106, CKA 198, YGO 23.

Besleria solanoides Kunth

Latizal; bosque poco intervenido, rastrojo; As. L - E, Com. B - S. San Agustín, vereda Yarumal, Pitalito, vereda El Pencil, CKA 56, CKA 238.

Grossulariaceae (1 género 1 especie)

Escallonia paniculata (Ruiz \& Pav.) Roem. \& Schult.

Nombre vernáculo: "chilco"

Fustal; bosque poco intervenido; As. W - C. San Agustín, vereda Alto Canelo, YGO 128.

\section{Hamamelidaceae (1 género, 1 especie)} Matudaea colombiana Lozano.
Latizal, fustal; bosque poco intervenido; Com. H - A. Pitalito, vereda El Pencil, CKA 204.

Hippocastanaceae (1 género, 1 especie) Billia rosea (Planch. \& Linden) C. Ulloa \& Jørg.

Nombre vernáculo: "cariseco" "Maiz tostao" Brinzal, latizal, fustal; bosque poco intervenido, bosque intervenido; As. L - E, As W - C, As G - M, Com. H - A, Com. Q - W. San Agustín, veredas San Antonio, Alto Canelo, Yarumal, Los Alpes - Marbella, Pitalito, veredas El Cedro, El Porvenir, Acevedo, vereda Villa Fátima, CKA 32, YGO 47, YGO 117, YGO 229, YGO 420.

\section{Icacinaceae (1 género, 1 especie)}

Calatola costaricensis Standl.

Latizal; bosque poco intervenido; Com. $\mathrm{H}-\mathrm{A}$. Pitalito, vereda El Cedro, CKA 166.

\section{Juglandaceae (1 género, 1 especie)}

Alfaroa cf. williamsii Ant. Molina Brinzal, latizal, fustal; bosque poco intervenido, bosque intervenido, rastrojo; As. L - E, Com. H - A, Com. B - S, Com. Q - W. Pitalito, veredas El Cedro, El Pencil, El Porvenir, Acevedo, vereda Villa Fátima, CKA 299, YGO 301, YGO 319, YGO 322.

\section{Lacistemataceae (1 género, 1 especie)}

Lozania mutisiana Schult.

Brinzal, fustal; bosque poco intervenido, bosque de regeneración; As. L-E, As G-M. San Agustín, veredas Yarumal, Villa Fátima, Los Alpes - Marbella, CKA 88, CKA 126.

\section{Lamiaceae (1 género, 2 especies)}

Hyptis atrorubens Poit.

Brinzal; rastrojo; As. G - M. San Agustín, vereda Marbella, YGO 280.

Hyptis pseudosinuata Epling

Brinzal; rastrojo; Com. B - S. San Agustín, veredas El Cedro, El Porvenir, YGO 358, YGO 390 


\section{Lauraceae (8 géneros, 23 especies)}

Aniba coto (Rusby) Kosterm.

Nombre vernáculo: "naranjuelo" "aguacatillo rojo"

Brinzal, latizal, fustal; bosque poco intervenido, bosque de regeneración; As. L - E, As. W - C, Com. Q - W. San Agustín, veredas Alto Canelo, Villa Fátima, Acevedo, vereda Villa Fátima, CKA 273, YGO 128, YGO 150, YGO 205, YGO 440, YGO 441.

\section{Aniba perutilis Hemsl.}

Nombre vernáculo: "medio comino"

Brinzal, latizal, fustal; bosque poco intervenido; Com. Q - W. Acevedo, vereda Villa Fátima, CKA 304, CKA 305, YGO 304.

Aniba cf. robusta (Klotzsch \& H. Karst.) Mez Nombre vernáculo: "aguacatillo" "laurel blanco" "aguacatillo blanco"

Brinzal, latizal, fustal; bosque poco intervenido, bosque de regeneración; As. L - E, As. W - C, As G - M, Com. H - A, Com Q - W. San Agustín, veredas San Antonio, Alto Canelo, Villa Fátima, Pitalito, vereda El Pencil, Acevedo, vereda Villa Fátima, CKA 73, CKA 318, YGO 6, YGO 374.

Beilschmiedia pendula (Sw.) Hemsl.

Nombre vernáculo: "aguacatillo" "laurel blanco"

Latizal, fustal; bosque poco intervenido; As. L - E, As. W - C. San Agustín, veredas San Antonio, Alto Canelo, YGO 111.

Beilschmiedia cf. tovarensis (Meisn.) Sahicko Nishida

Nombre vernáculo: "medio comino"

Latizal, fustal; bosque poco intervenido; As. L - E, Com. H - A. Pitalito, veredas El Pencil, El Porvenir, CKA 228.

Cinnamomum cf. triplinerve (Ruiz \& Pav.) Kosterm.
Nombre vernáculo: "aguacatillo" "aguacatillo rojo"

Brinzal, latizal, fustal; bosque poco intervenido; As. L - E, Com. H - A, Com. Q - W. San Agustín, vereda Yarumal, Pitalito, vereda El Cedro, Acevedo, vereda Villa Fátima, CKA 25, CKA 151, CKA 282, YGO 164, YGO 303 , YGO 325 .

Endlicheria formosa A.C. Sm.

Fustal; bosque poco intervenido; Com. $\mathrm{H}-\mathrm{A}$. Pitalito, vereda El Cedro, CKA 188.

Endlicheria cf. griseo-sericea Chanderbali Nombre vernáculo: "jigua" "chirimoyo" "medio comino"

Brinzal, latizal, fustal; bosque poco intervenido, bosque intervenido, rastrojo; As. L-E, As. $\mathrm{W}-\mathrm{C}$, As. G - M, Com. H - A, Com. B - A, Com. Q - W. San Agustín, veredas San Antonio, Los Alpes - Marbella, Pitalito, vereda El Cedro, El Pencil, El Porvenir, Acevedo, vereda Villa Fátima, CKA 21, CKA 118, CKA 216.

\section{Endlicheria sp.}

Latizal, fustal; bosque poco intervenido; As. L - E, Com- H - A. San Agustín, vereda San Antonio, Pitalito, vereda El Cedro, YGO 32, YGO 320

Licaria applanata van der Werff

Latizal; bosque intervenido, rastrojo; As. L - E, As. G - M, Com. H - A. San Agustín, vereda Los Alpes - Marbella, Pitalito, vereda El Porvenir, CKA 151a, CKA 261, YGO 234.

Nectandra cf. discolor (Kunth) Nees Nombre vernáculo: "laurel amarillo" Latizal, fustal; bosque poco intervenido, bosque intervenido, bosque de regeneración; As. L - E, As. G - M, Com. H - A. San Agustín, veredas Villa Fátima, Los Alpes - Marbella, Pitalito, vereda El Pencil, CKA 86, YGO 261.

Nectandra aff. globosa (Aubl.) Mez.

Nombre vernáculo: "medio comino" 
Latizal, fustal; bosque poco intervenido, bosque de regeneración; As. L- E, As. W-C. San Agustín, veredas Alto Canelo, Yarumal, Villa Fátima, CKA 83.

Nectandra cf. hihua (Ruiz. \& Pav.) Rohwer Nombre vernáculo: "laurel"

Brinzal, latizal, fustal; bosque poco intervenido, bosque intervenido, rastrojo; As. L-E, As. G - M, Com. H - A, Com. B - S. San Agustín, vereda Los Alpes - Marbella, Pitalito, veredas El Pencil, El Porvenir, CKA 233, CKA 242, CKA 272, YGO 242, YGO 386.

\section{Nectandra sp.}

Latizal; bosque poco intervenido; Com Q - W. Acevedo, vereda Villa Fátima, CKA 277.

Ocotea cf. floribunda (Sw.) Mez

Fustal; bosque poco intervenido; As. W - C. San Agustín, vereda Alto Canelo, YGO 147.

Ocotea macropoda (Kunth) Mez

Fustal; bosque poco intervenido, bosque de regeneración; As. L - E, Com. H - A. San Agustín, vereda Villa Fátima, Pitalito, vereda El Pencil, CKA 71, CKA 223.

Ocotea oblonga (Meisn.) Mez

Nombre vernáculo: "chaquiro"

Brinzal, latizal, fustal; bosque poco intervenido, bosque de regeneración; As. L - E, As. W - C. San Agustín, veredas San Antonio, Alto Canelo, YGO 31, YGO 68, YGO 202.

Ocotea cf. puberula (Rich.) Nees

Brinzal; bosque poco intervenido; Com. Q W. Acevedo, vereda Villa Fátima, YGO 429.

Ocotea smithiana $\mathrm{O}$. Schmidt

Brinzal, latizal; bosque poco intervenido; Com. Q - W. Acevedo, vereda Villa Fátima, CKA 283.

Ocotea tessmannii O. Schmidt

Nombre vernáculo: "tablero"
Brinzal, latizal; bosque intervenido, rastrojo; As. L - E. San Agustín, vereda Los Alpes - Marbella, Pitalito, veredas El Pencil, El Porvenir, YGO 260.

Ocotea sp.

Nombre vernáculo: "mulato"

Latizal, fustal; bosque poco intervenido, bosque de regeneración; As. L - E, Com $\mathrm{H}-\mathrm{A}$. San Agustín, vereda Villa Fátima, Pitalito, vereda El Cedro, CKA 218.

Persea rigens C.K. Allen

Latizal, fustal; bosque poco intervenido; As. L - E, Com. H - A. San Agustín, vereda San Antonio, Pitalito, vereda El Pencil, CKA 226, YGO 380.

Persea subcordata (Ruiz \& Pav.) Nees

Brinzal, fustal; bosque poco intervenido; As. L - E. San Agustín, vereda Yarumal, CKA 15, CKA 24.

\section{Lecythidaceae (1 género, 1 especie)}

Eschweilera sessilis A. C. Sm.

Fustal; bosque poco intervenido; Com. $\mathrm{H}-\mathrm{A}$. Pitalito, vereda El Cedro, CKA 189.

\section{Lythraceae (1 género, 1 especie)}

Cuphea racemosa (L. f.) Spreng.

Brinzal. rastrojo; As. G - M. San Agustín, vereda Marbella, YGO 276.

\section{Magnoliaceae (1 género, 2 especies)}

Dugandiodendron colombianum (Little) Lozano

Nombre vernáculo: "cobre"

Fustal; bosque poco intervenido; Com. $\mathrm{H}-\mathrm{A}$, Com. Q - W. Pitalito, veredas El Cedro, El Pencil, Acevedo, vereda Villa Fátima, CKA 179, CKA 307.

Dugandiodendron striatifolium (Little) Lozano Nombre vernáculo: "cobre" 
Brinzal, latizal; bosque poco intervenido; Com. Q - W. Acevedo, vereda Villa Fátima, CKA 275, CKA 312.

\section{Malpighiaceae (1 género, 1 especie)}

Stigmaphyllon bogotense Triana \& Planch.

Brinzal; rastrojo; As. G - M. San Agustín, vereda Marbella, YGO 296.

\section{Malvaceae (1 género, 1 especie)}

Sida rhombifolia $\mathrm{L}$.

Latizal; bosque poco intervenido; Com. B - S. Pitalito, vereda El Porvenir, CKA 252.

\section{Melastomataceae ( 7 géneros, 23 especies)}

Blakea calyptrata Gleason

Nombre vernáculo: "morochillo"

Latizal; bosque poco intervenido; Com. Q - W. Acevedo, vereda Villa Fátima, YGO 444.

Centronia eximia (Naudin) Triana

Latizal; bosque poco intervenido; As. L - E. San Agustín, vereda San Antonio, YGO 29.

Leandra cf. subseriata (Naudin) Cogn.

Latizal; rastrojo; As. G - M. San Agustín, vereda Los Alpes, YGO 291.

Meriania huilensis Wurdack

Latizal, fustal; Com. H - A. Pitalito, vereda El Cedro, CKA 169.

Meriania phlomoides (Triana) F. Almeda

Latizal; Com. H - A. Pitalito, vereda El Cedro, CKA 169a.

Miconia aeruginosa Naudin

Latizal; bosque poco intervenido; Com. Q - W. Acevedo, vereda Villa Fátima, CKA 293.

Miconia asperrima Triana

Latizal; bosque poco intervenido; Com. W-C. San Agustín, vereda Alto Canelo, YGO 118.

Miconia caudata (Bonpl.) DC.

Latizal; rastrojo; Com. B - S. Pitalito, vereda

El Pencil, CKA 241.
Miconia cf. costaricensis Cogn.

Brinzal, latizal; bosque poco intervenido, bosque intervenido, rastrojo; As. L-E, As. W - C, Com. B - S, Com. Q - W. San Agustín, vereda Alto Canelo, Pitalito, vereda El Porvenir, Acevedo, vereda Villa Fátima, CKA 279, CKA 321, YGO 52, YGO 404.

Miconia cf. dolichopoda Naudin

Nombre vernáculo: "morochillo rojo"

Brinzal, latizal, fustal; bosque poco intervenido, bosque intervenido, bosque de regeneración, rastrojo; As. L - E, As. G - M, Com. H - A, Com. Q - W. San Agustín, veredas Yarumal, Villa Fátima, Pitalito, veredas El Cedro, El Porvenir, Acevedo, vereda Villa Fátima, CKA 30, CKA 143, CKA 319, YGO 208, YGO 216, YGO 300.

Miconia lehmannii Cogn.

Latizal; bosque poco intervenido; As. W - C. San Agustin, vereda Alto Canelo, YGO 81.

Miconia aff. minuta Gleason

Brinzal, latizal; bosque intervenido; Com. B - S. Pitalito, veredas El Cedro, El Porvenir, CKA 199, YGO 395.

Miconia cf. pergamentacea Cogn. Latizal; bosque poco intervenido; Com. Q - W. Acevedo, vereda Villa Fátima, CKA 313.

Miconia psychrophila Naudin

Brinzal; bosque poco intervenido; As. L - E. San Agustín, vereda Yarumal, CKA 9.

Miconia resima Naudin

Brinzal, latizal, fustal; bosque poco intervenido, bosque intervenido, bosque de regeneración; As. L-E, As. W - C. San Agustín, veredas Alto Canelo, Yarumal, Los Alpes - Marbella, Pitalito, vereda El Porvenir, Acevedo, vereda Villa Fátima, CKA 6, CKA 78, CKA 97, YGO 390, YGO 414. 
Miconia cf. seposita Wurdack

Latizal; bosque poco intervenido; Com. Q - W. Acevedo, vereda Villa Fátima, CKA 291.

Miconia smaragdina Naudin

Nombre vernáculo: "morochillo"

Latizal; bosque intervenido; As. G - M. San Agustín, vereda Los Alpes -Marbella, YGO 264.

Miconia cf. theaezans (Bonpl.) Cogn.

Brinzal, fustal; bosque poco intervenido, bosque intervenido; As. L - E, Com. H - A. Pitalito, veredas El Cedro, El Porvenir, YGO 307.

Miconia cf. wurdackii L. Uribe

Brinzal, latizal; bosque poco intervenido; As. W - C. San Agustin, vereda Alto Canelo, YGO 57, YGO 96.

Miconia sp.

Latizal, fustal; bosque poco intervenido, bosque intervenido, rastrojo; As. L-E, As. W-C, As. G - M, Com. H - A. San Agustín, veredas Alto Canelo, Yarumal, Los Alpes - Marbella, Pitalito, veredas El Cedro, El Pencil, El Porvenir, CKA 34, CKA 123, CKA 231.

Monochaetum lineatum (D. Don) Naudin Brinzal; rastrojo; As. G - M. San Agustín, vereda Los Alpes, YGO 287.

Tibouchina ciliaris (Vent.) Cogn.

Brinzal; rastrojo; As. G - M, Com. B - S. San Agustín, vereda Marbella, Pitalito, vereda El Porvenir, YGO 274.

Tibouchina lepidota (Bonpl.) Baill.

Nombre vernáculo: "mayo"

Fustal; bosque de regeneración; As. L - E. San

Agustin, vereda Villa Fátima, CKA 81.

\section{Meliaceae ( 2 géneros, 3 especies)}

Guarea cf. kunthiana A. Juss.

Nombre vernáculo: "colorao"
Fustal; bosque poco intervenido; As. L - E. San Agustín, vereda San Antonio, YGO 11.

Ruagea tomentosa Cuatrec.

Latizal; bosque intervenido; As. L - E, As. G M. San Agustín, vereda Los Alpes - Marbella, Pitalito, vereda El Porvenir, CKA 134, CKA 263.

Ruagea sp.

Nombre vernáculo: "cedrillo"

Fustal; bosque poco intervenido; As. W - C, As. L - E. San Agustín, veredas San Antonio, Alto Canelo, YGO 19.

\section{Mimosaceae ( 2 géneros, 3 especies)}

Abarema cf. killipii (Britton \& Rose) Barneby \& J.W. Grimes

Fustal; bosque poco intervenido. Com. Q - W. Acevedo, vereda Villa Fátima, CKA 299.

Inga aff. acreana Harms

Latizal; bosque intervenido; As. G - M. San Agustín, vereda Los Alpes - Marbella, CKA 130.

Inga setosa $\mathrm{G}$. Don

Brinzal, latizal, fustal; bosque poco intervenido, bosque intervenido; As. L- E, Com. $\mathrm{H}-\mathrm{A}$. Pitalito, veredas El Cedro, El Porvenir, CKA 195, CKA 258.

\section{Monimiaceae (2 géneros, 4 especies)}

Mollinedia cf. repanda Ruiz \& Pav.

Brinzal, latizal, fustal; bosque poco intervenido, bosque intervenido; As. L - E, As. W - C, As. G-M, Com. H - A. San Agustín, veredas Alto Canelo, Los Alpes - Marbella, Pitalito, vereda El Cedro, CKA 55, CKA 122, YGO 109, YGO 140, YGO 144.

Mollinedia cf. tomentosa (Benth.) Tul. Nombre vernáculo: "arrayán guayabo" 
Latizal, fustal; bosque poco intervenido, bosque de regeneración; As. L-E, As. W - C, Com. Q - W. San Agustín, vereda San Antonio, Alto Canelo, Villa Fátima, CKA 294, YGO 10, YGO 132.

Siparuna grandiflora (Kunth) Perkins

Nombre vernáculo: "chilco blanco"

Brinzal; bosque poco intervenido, rastrojo; As. L - E, Com. B - S. San Agustín, vereda Yarumal, Pitalito, vereda El Pencil, CKA 8, YGO 391, YGO 392.

Siparuna lepidota (Kunth) A. DC.

Latizal; bosque poco intervenido; As. L - E. Pitalito, vereda El Cedro, CKA 68.

\section{Moraceae (3 géneros, 7 especies)}

Ficus caldasiana Dugand

Latizal, fustal; bosque poco intervenido, bosque intervenido, bosque de regeneración; As. L - E, As. G - M, Com. H - A. San Agustín, veredas Villa Fátima, Los Alpes - Marbella, Pitalito, vereda El Cedro, CKA 104.

Ficus mutisii Dugand

Latizal; rastrojo; As. G - M. San Agustín, Los Alpes - Marbella, YGO 262.

Ficus popayanensis Standl.

Fustal; bosque intervenido; As. L - E. Pitalito, vereda El Porvenir, CKA 266.

Ficus subandina Dugand

Fustal; bosque poco intervenido; Com. $\mathrm{H}-\mathrm{A}$. Pitalito, vereda El Cedro, CKA 194.

Ficus tonduzii Standl.

Latizal; bosque poco intervenido; As. L - E. San Agustín, vereda Yarumal, CKA 53.

Helicostylis tovarensis (Klotzsch \& H. Karst.) C.C. Berg

Brinzal, latizal, fustal; bosque poco intervenido, bosque intervenido, bosque de regeneración, rastrojo; As. $\mathrm{L}-\mathrm{E}$, As. $\mathrm{G}-\mathrm{M}$, Com.
H - A. San Agustín, veredas Villa Fátima, Los Alpes - Marbella, Pitalito, veredas El Cedro, El Pencil, El Porvenir, CKA 77, CKA 96, CKA 173, CKA 260, CKA 270, YGO 207.

Morus insignis Bureau

Nombre vernáculo: "lechero"

Brinzal, latizal, fustal; bosque poco intervenido, bosque intervenido, rastrojo; As. L - E, As. G - M, Com. B - S. San Agustín, veredas Yarumal, Los Alpes - Marbella, Pitalito, vereda El Cedro, CKA 31, CKA 254, YGO 341.

\section{Myrsinaceae (3 géneros, 9 especies)}

Cybianthus cuatrecasasii Pipoly

Brinzal, latizal, fustal; bosque poco intervenido; As. L-E, As W - C, Com. H - A. San Agustín, veredas San Antonio, Alto Canelo, Pitalito, vereda El Pencil, YGO 110, YGO 368.

Cybianthus iteoides (Benth.) G. Agostini

Latizal; bosque poco intervenido; As. W - C. San Agustín, vereda Alto Canelo, YGO 79.

Cybianthus cf. occigranatensis (Cuatrec.) G. Agostini

Brinzal, latizal, fustal; bosque poco intervenido; Com. Q - W. Acevedo, vereda Villa Fátima, CKA 325.

Cybianthus pastensis (Mez) G. Agostini Brinzal. bosque intervenido; As. G - M. San Agustín, vereda Los Alpes - Marbella, YGO 245.

Cybianthus cf. spichigeri Pipoly

Nombre vernáculo: "garrucho"

Brinzal, latizal, fustal; bosque poco intervenido, bosque intervenido, bosque de regeneración; As. L-E, Com. H - A, Com. Q - W. San Agustín, vereda Villa Fátima, Pitalito, veredas El Cedro, El Porvenir, Acevedo, vereda Villa Fátima, CKA 103. 
Cybianthus sp.

Brinzal, latizal; bosque poco intervenido; Com. H - A, Com. Q - W. Pitalito, vereda El Pencil, Acevedo, vereda Villa Fátima, CKA 324, YGO 367.

Geissanthus bogotensis Mez

Fustal; bosque poco intervenido; As. W - C. San Agustín, vereda Alto Canelo, YGO 156.

Myrsine guianensis (Aubl.) Kuntze

Fustal; bosque poco intervenido, bosque intervenido; As. G - M, Com. H - A. San Agustín, vereda Los Alpes - Marbella, Pitalito, vereda El Cedro, CKA 184.

Myrsine cf. pellucido-punctata Oerst.

Brinzal, latizal; bosque poco intervenido; Com. H - A. Pitalito, vereda El Pencil, YGO 366.

\section{Myrtaceae (1 género, 1 especie)}

Myrcianthes $\mathrm{sp}$.

Fustal; bosque poco intervenido; As. W - C. San Agustín, vereda Alto Canelo, YGO 154.

Piperaceae (2 géneros, 10 especies)

Peperomia aff. acuminata Ruiz \& Pav.

Brinzal; bosque poco intervenido; As. W - C. San Agustín, vereda Alto Canelo, YGO 101.

Peperomia angularis C. DC.

Brinzal; bosque poco intervenido; As L - E, As W - C. San Agustín, vereda Yarumal, CKA 45, YGO 174

Piper aff. aequale Vahl Brinzal; rastrojo; Com. B - S. Pitalito, vereda El Porvenir, CKA 255.

Piper caucaense Yunck.

Latizal; bosque poco intervenido; Com. $\mathrm{H}-\mathrm{A}$. Pitalito, vereda El Pencil, YGO 379.
Piper dichroostachyum Trel. \& Yunck.

Brinzal, latizal; bosque poco intervenido; As. L - E, Com. H - A. Pitalito, vereda El Cedro, YGO 162.

Piper lanceifolium Kunth Brinzal, latizal; rastrojo; Com. B - S. Pitalito, veredas El Cedro, El Pencil, CKA 212, YGO 352.

Piper marequitense C. DC.

Brinzal, latizal; rastrojo; Com. B - S. Pitalito, vereda El Pencil, YGO 355.

Piper aff. obliquum Ruiz \& Pav. Brinzal, latizal; bosque poco intervenido; As. L - E, Com. H - A. Pitalito, vereda El Pencil, YGO 371.

Piper subglabribracteatum C. DC.

Latizal; bosque poco intervenido; As. L - E. San Agustín, vereda San Antonio, YGO 34.

Piper aff. trigonum C. DC.

Brinzal, latizal; bosque poco intervenido, rastrojo; As. L - E, Com. B - S, Com. H - A. Pitalito, vereda El Porvenir, CKA 259.

\section{Polygalaceae (1 género, 1 especie)}

Monnina fastigiata Bonpl.

Latizal; bosque poco intervenido; Pitalito, vereda El Pencil, YGO 407.

\section{Proteaceae (1 género, 1 especie)}

Panopsis suaveolens (Klotzsch \& H. Karst.) Pittier

Fustal; bosque poco intervenido; As. W - C. San Agustín, vereda Alto Canelo, YGO 125.

\section{Rosaceae (3 géneros, 5 especies)} Hesperomeles obtusifolia (Pers.) Lindl. Fustal; bosque poco intervenido; As. W - C. San Agustín, vereda Alto Canelo, YGO 129. 
Prunus opaca (Benth.) Walp.

Latizal, fustal; bosque poco intervenido; As L - E, As W - C, Com. H - A. Pitalito, vereda El Cedro, San Agustín, vereda Alto Canelo, CKA 185, YGO 120.

\section{Prunus urotaenia Koehne}

Brinzal; bosque intervenido; As. G - M. San Agustín, vereda Los Alpes, YGO 241.

\section{Rubus cf. lechleri Focke}

Brinzal; bosque poco intervenido, bosque intervenido, rastrojo; As. G- M, As. L- E, Com. B - S. San Agustín, vereda Los Alpes, Pitalito, vereda El Cedro, YGO 199.

Rubus urticifolius Poir.

Latizal; rastrojo; As. G - M, Com. B - S. San Agustín, vereda Marbella, CKA 138.

\section{Rubiaceae (15 géneros, 28 especies)}

Cinchona lancifolia Mutis Brinzal, latizal, fustal; bosque poco intervenido, bosque intervenido; As. G - M, As. L - E, As. W - C. San Agustín, veredas Alto Canelo, San Antonio, vereda Yarumal, YGO 2, YGO 53, YGO 155, YGO 159, YGO 198.

Coccocypselum lanceolatum (Ruiz \& Pav.) Pers.

Brinzal; rastrojo; As. G - M. San Agustín, vereda Marbella, YGO 272.

Faramea flavicans (Kunth ex Roem. \& Schult.) Standl.

Brinzal, latizal, fustal; bosque poco intervenido; As. L- E, As. W - C, Com. Q - W. Acevedo, vereda Villa Fátima, San Agustín, veredas San Antonio, Alto Canelo, Villa Fátima, CKA 292, CKA 320, YGO 1, YGO 83, YGO 94, YGO 116, YGO 206.

Faramea multiflora A. Rich. ex DC. var. maynensis (Spruce ex Rusby) Steyerm.
Brinzal, latizal; bosque poco intervenido, bosque intervenido; As. G - M, Com. H - A. San Agustín, vereda Los Alpes, Pitalito, vereda El Pencil, CKA 236, YGO 236, YGO 258.

Elaeagia myriantha (Standl.) C. M. Taylor \& Hammel

Nombre vernáculo: "aguacatillo" "colorao" "huesillo"

Brinzal, latizal, fustal; bosque poco intervenido, bosque intervenido, bosque de regeneración; As. L - E, As. W - C, As. G - M, Com. Q - W. San Agustín, veredas San Antonio, Alto Canelo, Yarumal, Villa Fátima, Los Alpes - Marbella, CKA 18, CKA 27, CKA 315, YGO 4, YGO 13, YGO 131, YGO 256.

Galium hypocarpium Endl. ex Griseb.

Brinzal; rastrojo; As. G - M. San Agustín, vereda Marbella, YGO 278.

Guettarda crispiflora Vahl subsp. sabiceoides (Standl.) C.M. Taylor

Latizal; bosque intervenido, rastrojo; As. G - M, Com. B - S. San Agustín, vereda Los Alpes, Pitalito, vereda El Pencil, YG0 263, YGO 363.

Guettarda hirsuta Pers.

Latizal, fustal; bosque intervenido, rastrojo; As. G - M, Com. B - S. San Agustín, veredas Los Alpes, Marbella, Pitalito, vereda El Pencil, CKA 124, CKA 206.

Hillia parasitica Jacq.

Brinzal; bosque poco intervenido; As. L - E. San Agustín, vereda Villa Fátima, CKA 101.

Hoffmannia sprucei Standl.

Brinzal, latizal; bosque intervenido, rastrojo; As. G - M, As. L - E, Com. B - S, Com. H - A. Pitalito, veredas El Cedro, El Porvenir, San Agustín, vereda Yarumal, CKA 136, YGO 166, YGO 349, YGO 401. 
Ladenbergia macrocarpa (Vahl) Klotzsch

Latizal, fustal; bosque poco intervenido, bosque intervenido; As. G - M, As. L - E. San Agustín, veredas San Antonio, Villa Fátima, Pitalito, vereda El Porvenir, CKA 75, CKA 267, YGO 3.

Notopleura macrophylla (Ruiz \& Pav.) C.M. Taylor

Brinzal; bosque poco intervenido, rastrojo; Com. B - S, Com. H - A. Pitalito, vereda El Porvenir, YGO 259, YGO 405.

Palicourea acetosoides Wernham

Brinzal; bosque poco intervenido; As. L - E. Pitalito, vereda El Porvenir, YGO 418.

Palicourea angustifolia Kunth

Brinzal, latizal, fustal; bosque poco intervenido, bosque intervenido; As. G - M, As. L-E, As. W - C, Com. Q - W. Acevedo, vereda Villa Fátima, San Agustín, veredas Alto Canelo, Villa Fátima, CKA 125, CKA 302, CKA 328, YGO 82b, YGO 85, YGO 191.

Palicourea cf. apicata Kunth

Brinzal; bosque poco intervenido; As. W - C. San Agustín, vereda Alto Canelo, YGO 80.

Palicourea calophlebia Standl.

Brinzal, latizal; bosque poco intervenido, bosque intervenido, rastrojo; As. G - M, As. L - E, Com. B - S, Com. H - A, Com. Q - W. Acevedo, vereda Villa Fátima, San Agustín, veredas Los Alpes, Villa Fátima, Pitalito, veredas El Cedro, El Pencil, El Porvenir, CKA 98, CKA 244, CKA 257, YGO 218, YGO 225, YGO 357, YGO 364, YGO 437.

Palicourea cuatrecasasii Standl. Ex Steyerm. Brinzal, latizal, fustal; bosque poco intervenido, bosque intervenido; As. G - M, As. L-E, As. W - C. San Agustín, veredas Alto Cane1o, Los Alpes, San Antonio, Villa Fátima, Yarumal, CKA 3, CKA 94, CKA 132, YGO 14,
YGO 24, YGO 25, YGO 27, YGO 50, YGO 54, YGO 175, YGO 192.

Palicourea cf. demissa Standl.

Brinzal; bosque poco intervenido; Com. $\mathrm{H}-\mathrm{A}$. Pitalito, vereda El Cedro, YGO 310.

Palicourea dorantha Wernham Latizal; bosque poco intervenido; Com. $\mathrm{H}-\mathrm{A}$. Pitalito, vereda El Cedro, YGO 298.

Palicourea cf. garciae Standl.

Brinzal, latizal, fustal; bosque poco intervenido, bosque intervenido; As. G - M, As. L - E, Com. H - A. Pitalito, veredas El Cedro, El Pencil, El Porvenir, CKA 148, CKA 224, CKA 271.

\section{Palicourea sp.}

Fustal; bosque poco intervenido; As. L - E. San Agustín, vereda Yarumal, CKA 29.

Posoqueria velutina Standl.

Latizal; bosque intervenido; As. G - M. San Agustín, vereda Los Alpes, YGO 255.

Psychotria aschersoniana K. Schum. \& Krause

Brinzal; bosque poco intervenido, bosque intervenido, rastrojo; As. L - E, Com. B - S, Com. H - A. Pitalito, veredas El Cedro, El Porvenir, YGO 195, YGO 196, YGO 346, YGO 410, YGO 417, YGO 419.

Psychotria cf. acuminata Benth.

Brinzal, latizal; bosque poco intervenido, bosque intervenido; As. L-E, Com. H - A. Pitalito, vereda El Pencil, YGO 373.

Psychotria bracteata DC.

Brinzal, latizal; bosque poco intervenido, bosque intervenido; As. G - M, As. L - E. San Agustín, veredas Los Alpes, Yarumal, CKA 42, YGO 227. 
Psychotria longirostris (Rusby) Standl.

Latizal, fustal; bosque poco intervenido. Com. H - A. Pitalito, vereda El Pencil, CKA 232.

\section{Rudgea sp.}

Brinzal, fustal; bosque poco intervenido, bosque intervenido, bosque de regeneración, rastrojo; As. G - M, As. L - E, As. W - C, Com. B - S, Com. Q - W. Acevedo, vereda Villa Fátima, San Agustín, veredas Alto Canelo, Los Alpes, San Antonio, Pitalito, vereda El Porvenir, CKA 298, YGO 5, YGO 143, YGO 226, YGO 413.

Tocoyena costanensis Steyerm.

Brinzal, latizal, fustal; bosque poco intervenido, bosque intervenido; As. G - M, As. W - C. San Agustín, veredas Alto Canelo, Los Alpes, CKA 39, YGO 134, YGO 267.

\section{Sabiaceae (1 género, 2 especies)}

Meliosma aff. bogotana Steyerm.

Latizal; bosque intervenido; As. G - M. San

Agustín, vereda Los Alpes, YGO 246.

Meliosma violacea Cuatrec. \& Idrobo

Fustal; bosque poco intervenido; As. W - C. San Agustín, vereda Alto Canelo, YG 80.

\section{Sapindaceae ( 3 géneros, 3 especies)}

Cupania aff. americana $\mathrm{L}$.

Latizal; bosque intervenido; As. G - M. San Agustín, vereda Los Alpes, YGO 257.

Matayba cf. arborescens (Aubl.) Radlk.

Fustal; bosque poco intervenido; Com. $\mathrm{H}-\mathrm{A}$. Pitalito, vereda El Cedro, YGO 313.

Paullinia sp.

Brinzal; bosque intervenido; As. G - M. San Agustín, vereda Los Alpes, YGO 240.

Sapotaceae ( 2 géneros, 3 especies)

Chrysophyllum argenteum subsp. auratum (Miq.) T. D. Penn.
Latizal, fustal; bosque de regeneración; Com. Q - W. Acevedo, vereda Villa Fátima, CKA 284.

Pouteria baehniana Monach.

Latizal, fustal; bosque poco intervenido; As. W - C, Com. H - A. Pitalito, veredas El Cedro, El Pencil, San Agustín, veredas Alto Canelo, CKA 175, CKA 219, CKA 222, YGO 139.

Pouteria torta (Mart.) Radlk.

Brinzal, latizal, fustal; bosque poco intervenido, bosque de regeneración; Com. $\mathrm{H}-\mathrm{A}$, Com. Q - W. Acevedo, vereda Villa Fátima, CKA 276, CKA 297b, YGO 445.

\section{Simaroubaceae (1 género, 1 especie)}

Picramnia sphaerocarpa Planch.

Brinzal; bosque poco intervenido; As. G - M, Com. H - A. Pitalito, vereda El Cedro, YGO 333.

\section{Solanaceae ( 3 géneros, 5 especies)}

Cuatresia riparia (Kunth) Hunz.

Brinzal, latizal; bosque poco intervenido; As. L - E, Com. H - A. San Agustín, vereda San Antonio, Pitalito, vereda El Pencil, YGO 36, YGO 372.

Lycianthes inaequilatera Bitter

Brinzal; bosque poco intervenido; As. L - E. San Agustín, vereda Yarumal, YGO 171.

Solanum acerifolium Humb. \& Bonpl. ex Dun.

Latizal; rastrojo; As. G - M. San Agustín, vereda Marbella, CKA 139.

Solanum deflexiflorum Bitter

Fustal; bosque poco intervenido; As. W - C. San Agustín, vereda Alto Canelo, YGO 137.

Solanum aff. subinerme Jacq.

Latizal; rastrojo; Com. B - S. Pitalito, vereda El Porvenir, CKA 253. 
Staphyleaceae ( 2 géneros, 2 especies)

Huertea glandulosa Ruiz \& Pav.

Latizal; bosque intervenido; As. G - M. San

Agustín, vereda Los Alpes, YGO 252.

Turpinia occidentalis (Sw.) G. Don

Fustal; bosque poco intervenido; As. L - E. San Agustín, vereda San Antonio, YGO 17.

\section{Styracaceae (1 género, 1 especie)}

Styrax aff. pentlandianus J. Rémy

Latizal; bosque de regeneración; Com. Q - W.

Acevedo, vereda Villa Fátima, YGO 426.

\section{Symplocaceae (1 género, 1 especie)}

Symplocos aff. flosfragrans Chaparro

Fustal; bosque poco intervenido; As. W - C.

San Agustín, vereda Alto Canelo, YGO 158.

\section{Theaceae (3 géneros, 3 especies)}

Freziera cf. karsteniana (Szyszyl.) Kobuski

Fustal; bosque poco intervenido; As. W - C. San Agustín, vereda Alto Canelo, YGO 151.

Gordonia cf. fruticosa (Schrad.) H. Keng

Latizal; bosque intervenido; As. L- E. Pitalito, vereda El Porvenir, YGO 408.

Ternstroemia cf. macrocarpa Triana \& Planch. Fustal; bosque de regeneración; Com. Q - W. Acevedo, vereda Villa Fátima, YGO 443.

\section{Tiliaceae (1 género, 1 especie)}

Heliocarpus americanus L.

Brinzal, latizal, fustal; bosque poco intervenido, bosque intervenido, rastrojo; As. G - M, Com. B - S, Com. H - A. Pitalito, vereda El Pencil, CKA 21.

\section{Ulmaceae (1 género, 1 especie)}

Lozanella enantiophylla (Donn. Sm.) Killip \& C. V. Morton

Fustal; bosque poco intervenido; As. L - E. San Agustín, vereda San Antonio, YGO 39a.

\section{Urticaceae (4 géneros, 5 especies)}

Boehmeria aspera Wedd.

Latizal; rastrojo; Com. B - S. Pitalito, vereda

El Pencil, YGO 360.

Boehmeria caudata Sw.

Brinzal, latizal; rastrojo; Com. B - S. Pitalito, vereda El Cedro, YGO 342, YGO 356.

Phenax cf. rugosa (Poir.)Wedd.

Latizal; rastrojo; Com. B - S. Pitalito, vereda El Pencil, YGO 362.

Pilea rhombea (L. f.) Liebm.

Brinzal; rastrojo; Com. B - S. Pitalito, vereda El Pencil, San Agustín, vereda Marbella, YGO 286.

Urera caracasana Griseb.

Latizal; bosque intervenido; As. L - E. Pitalito, vereda El Porvenir, CKA 262.

\section{Verbenaceae (1 género, 2 especies)}

Aegiphila aff. membranacea Turcz.

Latizal; bosque de regeneración; Com. Q - W. Acevedo, vereda Villa Fátima,YGO 434.

Aegiphila aff. mollis Kunth

Latizal; bosque poco intervenido; Com. $\mathrm{H}-\mathrm{A}$.

Pitalito, vereda El Cedro, CKA 168.

\section{Vitaceae (1 género, 1 especie)}

Cissus granulosa Ruiz \& Pav.

Brinzal; bosque poco intervenido; As. W - C. San Agustín, vereda Alto Canelo, YGO 90.

\section{Vochysiaceae (1 género, 1 especie)}

Vochysia aff. megalantha Stafleu

Fustal; bosque intervenido; As. G - M. San Agustín, vereda Los Alpes, CKA 142.

\section{Winteraceae (1 género, 1 especie)}

Drimys granadensis L.f.

Fustal; bosque intervenido; As. G - M. San Agustín, vereda Los Alpes, CKA 119. 


\section{MONOCOTILEDÓNEAS}

\section{Araceae (3 géneros, 6 especies)}

Anthurium cf. corrugatum Sodiro

Brinzal; bosque poco intervenido; As. L - E. San Agustín, vereda San Antonio, YGO 48.

Anthurium longegeniculatum Engl.

Brinzal, latizal; bosque poco intervenido, bosque intervenido, rastrojo; As. L - E, As. W - C, As. G - M, Com. H - A, Com. B - S. San Agustín, veredas Alto Canelo, Yarumal, Pitalito, vereda El Cedro, CKA 149, CKA 201, YGO 59, YGO 163, YGO 215.

Anthurium oxybelium Schott

Brinzal; bosque poco intervenido; As. L - E, Com. H - A. San Agustín, vereda Yarumal, Pitalito, vereda El Cedro, Acevedo, vereda Villa Fátima, CKA 4, CKA 157.

Philodendron cf. gloriosum Andre

Latizal; rastrojo; Com. B - S. Pitalito, veredas El Cedro, El Pencil, CKA 197, YGO 395.

\section{Philodendron sp.}

Nombre vernáculo: "gramalote"

Brinzal; bosque poco intervenido, bosque intervenido; As. L - E, As. W - C, As. G - M. San Agustín, veredas Alto Canelo, Yarumal, Los Alpes, CKA 1, YGO 104, YGO 393.

\section{Syngonium podophyllum Schott}

Brinzal; bosque poco intervenido; As. L - E. San Agustín, vereda Yarumal, YGO 170.

\section{Arecaceae (6 géneros, 8 especies)}

Aiphanes cf. linearis Burret

Nombre vernáculo: "palma osa" "palma chonta"

Latizal, fustal; bosque poco intervenido; Com. Q - W. Acevedo, vereda Villa Fátima, YGO 427.

Chamaedorea pinnatifrons (Jacq.) Oerst.

Brinzal, latizal; bosque intervenido, rastrojo; As. L - E, As. G - M, Com. B - S, Com. H
- A. San Agustín, veredas San Antonio, Yarumal, Villa Fátima, Pitalito, veredas El Cedro, El Pencil, El Porvenir, CKA 36, CKA 40, YGO 50, YGO 217a.

Dictyocaryum lamarckianum (Mart.) H. Wendl. Nombre vernáculo: "palma bombona"

Latizal, fustal; bosque poco intervenido; Com. H - A, Com. Q - W. Pitalito, vereda El Pencil, Acevedo, vereda Villa Fátima, CKA 217, CKA 295.

Geonoma cf. orbigniana Mart.

Nombre vernáculo: "palma San José"

Latizal; bosque poco intervenido; Com. $\mathrm{H}-\mathrm{A}$, Com. Q - W. Pitalito, vereda El Pencil, Acevedo, vereda Villa Fátima, YGO 388.

\section{Geonoma sp.}

Brinzal, latizal; bosque poco intervenido, bosque de regeneración; As. L - E, As W - C. San Agustín, veredas Alto Canelo, Yarumal, Villa Fátima, CKA 17, CKA 93, YGO 56.

Prestoea carderi (W. Bull) Hook. f.

Nombre vernáculo: "palmicha"

Brinzal, latizal, fustal; bosque poco intervenido; As. W - C, Com. H - A. San Agustín, vereda El Cedro, Pitalito, vereda El Pencil, YGO 382.

Wettinia fascicularis (Burret) H.E. Moore \& Dransf.

Nombre vernáculo: "palma corunta"

Brinzal, latizal, fustal; bosque poco intervenido; As. L - E, Com. Q - W. Pitalito, vereda El Porvenir, Acevedo, vereda Villa Fátima, CKA 217, CKA $217 b$.

\section{Wettinia sp.}

Nombre vernáculo: "galiche"

Brinzal, latizal, fustal; bosque poco intervenido, bosque intervenido; As. G - M, Com. H - A. San Agustín, vereda Los Alpes, Pitalito, vereda El Cedro, CKA 118, YGO 233. 


\section{Cyclanthaceae (1 género, 1 especie)}

Sphaeradenia sp.

Nombre vernáculo: "palma parquetilla"

Brinzal, latizal; bosque poco intervenido, rastrojo; As. L- E, As. G-M, Com. H - A, Com. B - S, Com. Q - W. San Agustín, vereda Villa Fátima, Pitalito, vereda El Pencil, Acevedo, vereda Villa Fátima, CKA 91, YGO 389.

\section{Heliconiaceae (1 género, 1 especie)}

Heliconia aff. burleana J. E. Abalo \& G. Morales $\mathrm{L}$.

Nombre vernáculo: "platanillo"

Brinzal, latizal; bosque intervenido, rastrojo; As. G - M, Com B - S. San Agustín, vereda
Los Alpes, Pitalito, vereda El Porvenir, YGO 223, YGO 407a.

\section{Smilacaceae (1 género, 1 especie)}

Smilax tomentosa Kunth

Brinzal; rastrojo; Com. B - S. Pitalito, vereda El Cedro, CKA 205.

\section{Zingiberaceae (1 género, 1 especie)}

Renealmia ligulata Maas

Latizal; bosque poco intervenido; As. L - E. San Agustín, vereda Yarumal, CKA 57. 